\title{
On Linear Differential Equations Involving a Para-Grassmann Variable
}

\author{
Toufik MANSOUR ${ }^{\dagger}$ and Matthias SCHORK ${ }^{\ddagger}$ \\ $\dagger$ Department of Mathematics, University of Haifa, 31905 Haifa, Israel \\ E-mail: toufik@math.haifa.ac.il \\ URL: http://www.math.haifa.ac.il/toufik/ \\ ¥Camillo-Sitte-Weg 25,60488 Frankfurt, Germany \\ E-mail: mschork@member.ams.org
}

Received May 01, 2009, in final form July 05, 2009; Published online July 15, 2009

doi:10.3842/SIGMA.2009.073

\begin{abstract}
As a first step towards a theory of differential equations involving paraGrassmann variables the linear equations with constant coefficients are discussed and solutions for equations of low order are given explicitly. A connection to $n$-generalized Fibonacci numbers is established. Several other classes of differential equations (systems of first order, equations with variable coefficients, nonlinear equations) are also considered and the analogies or differences to the usual ("bosonic") differential equations discussed.
\end{abstract}

Key words: para-Grassmann variables; linear differential equations

2000 Mathematics Subject Classification: 11B39; 13A99; 15A75; 34A30; 81R05; $81 \mathrm{~T} 60$

\section{Introduction}

Schwinger introduced anticommuting variables (i.e., Grassmann algebras) into physics in order to extend his treatment to fermions of quantum fields using Green's functions and sources [1]. A few year later Martin extended Feynman's path integral method of quantization to systems containing fermions and needed a "classical" fermion to quantize, thereby extending Schwinger's analysis of Grassmann algebras [2]. These anticommuting variables $\theta_{i}$ satisfying $\theta_{i} \theta_{j}=-\theta_{j} \theta_{i}-$ implying in particular that $\theta_{i}^{2}=0$ - were used by a number of authors to develop a systematic quantization of fermions in analogy to the well-known quantization of bosons based on the conventional commuting variables, see, e.g., the monograph of Berezin [3] for an early survey. Around 1970 the idea of a symmetry between bosons and fermions - called supersymmetry emerged (for accounts on the early history see, e.g., [4]), providing a link between particles having different statistics. It took then only a few years until the commuting and anticommuting variables were merged and interpreted as coordinates on a "superspace" (mainly due to Akulov and Volkov) and the physical superfields as "functions" on superspace (mainly due to Salam, Strathdee, Ferrara, Wess and Zumino). This happened in 1974 and from then on the idea of a physical and mathematical superworld has captured the imagination of physicists and mathematicians alike (see, e.g., [5, 6] for a physical discussion and [7, 8, 9] for a mathematical discussion). In our context it is particularly interesting to note that the introduction of the anticommuting (and nilpotent) variables has led to beautiful extensions of well-known mathematical structures (supermanifolds, supergroups, etc.).

In a rather different line of thought Green introduced in 1953 parastatistics into quantum field theory by considering certain trilinear commutation relations, generalizing the quantization schemes for bosons and fermions [10]. With hindsight it is not surprising what the next steps should have been in analogy to Fermi-Dirac statistics (i.e., fermions): The introduction of the 
"classical paraobjects" which yield upon quantization the operators satisfying the trilinear commutation relations (and are nilpotent in higher order). This should motivate the introduction of para-Grassmann algebras and then, in close analogy to above, a merging of commuting and para-Grassmann variables as coordinates on a parasuperspace and describing physical parasuperfields as functions on this "space". From a more mathematical point of view one would hope that these parastructures would again yield fascinating new extensions of well-known structures. In reality the development took some time; although there had been precursors $[2,11]$ the real development took off around 1979 with the work of Kamefuchi and coworkes [12, 13, 14]. In their work the focus was on the quantum field theory of particles satisfying parastatistics, see, e.g., the early monograph [15]. Para-Grassmann variables - satisfying $\left[\theta_{i},\left[\theta_{j}, \theta_{k}\right]\right]=0$ as well as $\left[\theta_{i_{1}}, \theta_{i_{2}}, \ldots, \theta_{i_{m}}\right]_{+}=0$ where $m \geq p+1$ and $[., \ldots, .]_{+}$denotes the completely symmetrized product (note that this implies $\theta_{i}^{p+1}=0$ ) - were mainly considered as a convenient tool, but already in [13] parasuperfields depending on conventional and para-Grassmann variables were introduced in close analogy to superfields. One of the first applications were in a model of one-dimensional supergravity interacting with "matter" represented by para-Grassmann-variables [16] and in a para-Grassmann extension of the Neveu-Schwarz-Ramond algebra [17]. Further applications have been in a parasupersymmetric extension of quantum mechanics [18, 19, 20, 21, 22], in particular in generalizations of the Pauli and Dirac equations [23, 24] to describe particles with spin $s>\frac{1}{2}$, as well as in the closely related fractional supersymmetry [25, 26, 27, 28, 29]. Low dimensional field theories, in particular conformal field theories, have also been discussed $[30,31,32,33,34,35,36,37,38,39]$ and a generalization of the Virasoro algebra has been discovered $[40,41]$. In particular in this approach the geometrical point of view of merging the ordinary variables with the para-Grassmann variables into a set of coordinates on a parasuperspace and considering the fractional supersymmetric fields as functions on this space has been used in close analogy to the well-known superspace and superfields. From a different point of view a connection to deformed algebras or quantum groups was reported [42, 43, 44, 45, 46, 47, 48, 49]. Of course, para-Grassmann variables have also been considered in their own right $[50,51,52,53]$. Many more references can be found in [54] which surveys also some of these developments.

Let us point out that the para-Grassmann variables considered originally (satisfying trilinear relations) lead quickly to rather messy structures and calculations. Therefore, a generalization of the Grassmann variables was introduced by Filippov, Isaev and Kurdikov in [41, 42, 51]

satisfying $\theta_{i}^{p+1}=0$ as well as certain bilinear commutation relations of the form $\theta_{i} \theta_{i}=e^{\frac{2 \pi i}{p+1}} \theta_{j} \theta_{i}$ (for $i<j$ ). In most of the more recent works (i.e., after 1992) these generalized Grassmann variables - also called para-Grassmann variables - are used. Of course, in the single-variable case these different approaches reduce to the same sole condition $\theta^{p+1}=0$ and the corresponding structure including a parasupercovariant derivative has been introduced in [30] (a formal variable $\lambda$ satisfying $\lambda^{p+1}=0$ was already considered by Martin in [2]).

From a more mathematical point of view one may hope that there exists an associated $\mathbf{Z}_{p+1^{-}}$ graded mathematics, yielding for $p=1$ "ordinary" supermathematics. Some results in this direction have already been obtained in the literature cited above. In particular, in the paper [53] by one of the authors many basic elements of para-Grassmann calculus (involving a single para-Grassmann variable) are collected and some simple observations concerning differential equations involving a para-Grassmann variable were obtained. It is the aim of the present paper to start a systematic treatment of differential equations involving para-Grassmann variables.

Before turning to a more detailed description of the present paper we would like to point out that in [48] a differential equation containing a para-Grassmann variable is solved in connection with deformed coherent states. To the best of our knowledge this is the only paper where such a differential equation is solved explicitly (more details can be found below).

Now, let us describe the contents of the present paper in more detail. In Section 2 the definitions and basic properties of para-Grassmann calculus are recalled and the notation is 
fixed. In Section 3 some of the simplest differential equations are solved and compared to the usual "bosonic" differential equations. The linear differential equations of second order (with constant coefficients) are considered in Section 4. In the direct approach chosen a recurrence relation has to be solved which is closely related to Fibonacci numbers. The results obtained are extended to linear differential equations of arbitrary order in Section 5. However, since the method used for the second order case turns out to be rather cumbersome in the general case (involving recurrence relations related to $n$-generalized Fibonacci numbers) we follow a slightly different approach. The main difficulty consists in getting control over the case where degeneracies appear in the associated characteristic equation. In Section 6 the general system of first order is considered. It is rather simple to describe the solution of this system abstractly but when considering concrete examples tedious calculations result, too. The peculiarities of the linear differential equation with variable coefficients are sketched in Section 7 by considering an example. In Section 8 a very simple nonlinear differential equation is treated. It turns out that in contrast to the linear differential equations where a close analogy to the "bosonic" case holds true - due to the nilpotency of the para-Grassmann variable the equations show a behaviour reminding one also of "bosonic" partial differential equations. Finally, some conclusions are presented in Section 9.

\section{Some formulae of para-Grassmann calculus}

In this section we briefly recall some basic facts about para-Grassmann calculus following [53] (see also $[30,42,41,51]$ ). For a given natural number $p \geq 1$ the nilpotent "coordinate" $\theta$ vanishes in $\left(p+1\right.$ )-th order, i.e., $\theta^{p+1}=0$ (with $\theta^{l} \neq 0$ for $0 \leq l \leq p$ ); the case $p=1$ reproduces the well known Grassmann variable appearing in supersymmetric theories. Due to our assumption there will be the linearly independent (over $\mathbb{C}$ ) elements $\left\{1, \theta, \theta^{2}, \ldots, \theta^{p}\right\}$. In analogy to "ordinary" supermathematics we introduce "parasuperfunctions" $f(x, \theta)$, which can be expanded as a finite series in $\theta$ :

$$
f(x, \theta)=f_{0}(x)+f_{1}(x) \theta+\cdots+f_{p}(x) \theta^{p} .
$$

The functions $f_{k}(x)$ will be called the components (or sometimes coefficients) of $f(x, \theta)$. We introduce a derivative $\partial \equiv \partial_{\theta}$ satisfying the following commutation relation:

$$
\partial \theta=q \theta \partial, \quad q=e^{\frac{2 \pi i}{p+1}} .
$$

In the following we use the standard notation

$$
[n]_{q}=1+q+\cdots+q^{n-1} \equiv \frac{1-q^{n}}{1-q}, \quad[n]_{q} !=[n]_{q}[n-1]_{q} \cdots[1]_{q}
$$

with $[0]_{q}=[0]_{q} !=1$. It is easy to check that the commutation relation $(2.2)$ implies $\partial \theta=1$, $\partial \theta^{2}=(1+q) \theta$, and in general

$$
\partial \theta^{n}=[n]_{q} \theta^{n-1} \text {. }
$$

Note that $\partial^{p+1}=0$. For parasuperfunctions we have (at least) two derivatives, the "normal" derivative $\partial_{x} \equiv \frac{d}{d x}$ and $\partial$ acting on the nilpotent variables. We introduce a parasupercovariant derivative by $[30,42,41,51]$

$$
\mathcal{D}:=\partial+\frac{\theta^{p}}{[p]_{q} !} \partial_{x}
$$


It is straightforward to check that for $f$ as in (2.1) one has (for $0 \leq r \leq p$ )

$$
\mathcal{D}^{r} f(x, \theta)=\sum_{k=0}^{r-1} \frac{[k]_{q} !}{[p-(r-1)+k]_{q} !} \partial_{x} f_{k}(x) \theta^{p-(r-1)+k}+\sum_{k=r}^{p} \frac{[k]_{q} !}{[k-r]_{q} !} f_{k}(x) \theta^{k-r} \text {. }
$$

Note in particular that the parasupercovariant derivative $\mathcal{D}$ is a $(p+1)$-th root of $\partial_{x}$ in the sense that $[30,42,41,51]$

$$
\mathcal{D}^{p+1} f(x, \theta)=\partial_{x} f(x, \theta) .
$$

Due to this relation there exists a certain "periodicity" in the derivatives $\mathcal{D}^{s} f$ of a function $f$ where now $s$ is an arbitrary integer. Since $s$ can be decomposed in a unique way as $s=$ $s^{\prime}(p+1)+s^{\prime \prime}$ with $0 \leq s^{\prime \prime}<p+1$, one may use (2.4) and (2.5) to obtain

$$
\begin{aligned}
\mathcal{D}^{s} f(x, \theta)= & \sum_{k=0}^{s^{\prime \prime}-1} \frac{[k]_{q} !}{\left[p-\left(s^{\prime \prime}-1\right)+k\right]_{q} !} \partial_{x}^{s^{\prime}+1} f_{k}(x) \theta^{p-\left(s^{\prime \prime}-1\right)+k} \\
& +\sum_{k=s^{\prime \prime}}^{p} \frac{[k]_{q} !}{\left[k-s^{\prime \prime}\right]_{q} !} \partial_{x}^{s^{\prime}} f_{k}(x) \theta^{k-s^{\prime \prime}}
\end{aligned}
$$

It is also possible to introduce a formal "antiderivative" by letting [50]

$$
\mathcal{D}^{-1} f(x, \theta):=[p]_{q} ! \int^{x} f_{p}(t) d t+f_{0}(x) \theta+\frac{1}{[2]_{q}} f_{1}(x) \theta^{2}+\cdots+\frac{1}{[p]_{q}} f_{p-1}(x) \theta^{p}
$$

so that $\mathcal{D}^{-1} \mathcal{D} f=f$; in particular, $\mathcal{D}^{-1} 1=\theta$. More generally, iterating this allows one to introduce an operator $\mathcal{D}^{-s}$. Let us also introduce an exponential function in a bosonic and a para-Grassmann variable by setting [42]

$$
e_{q}(x ; \theta):=e^{x} \sum_{n=0}^{p} \frac{\theta^{n}}{[n]_{q} !} .
$$

Then it is an easy calculation to verify that it has the defining property of the exponential function, i.e., $\mathcal{D} e_{q}(x ; \theta)=e_{q}(x ; \theta)$. Under a scaling of the arguments we obtain the following nice expression [42]:

$$
\mathcal{D} e_{q}\left(\beta^{p+1} x ; \beta \theta\right)=\beta e_{q}\left(\beta^{p+1} x ; \beta \theta\right) .
$$

This implies $\mathcal{D}^{p+1} e_{q}\left(\beta^{p+1} x ; \beta \theta\right)=\partial_{x} e_{q}\left(\beta^{p+1} x ; \beta \theta\right)$, as it should according to $(2.5)$.

\section{Some very simple differential equations}

In this brief section we consider some simple differential equations. Most of these results can be found already in [53] but for the convenience of the reader and the systematic exposition we have included the results here. Now, let us consider the very simple differential equation

$$
\mathcal{D}^{s} f(x, \theta)=0 .
$$

Writing $s=s^{\prime}(p+1)+s^{\prime \prime}$ with $0 \leq s^{\prime \prime}<p+1$, we may use (2.6) to obtain the following conditions on the components $f_{k}(x)$ :

$$
\partial_{x}^{s^{\prime}+1} f_{k}(x)=0,0 \leq k \leq s^{\prime \prime}-1, \quad \partial_{x}^{s^{\prime}} f_{k}(x)=0, s^{\prime \prime} \leq k \leq p .
$$


Thus, the first $s^{\prime \prime}$ components are polynomials of degree $s^{\prime}$, whereas the remaining components are polynomials of degree $s^{\prime}-1$. In particular, there are $s^{\prime \prime}\left(s^{\prime}+1\right)+\left(p+1-s^{\prime \prime}\right) s^{\prime}=s^{\prime}(p+1)+s^{\prime \prime} \equiv s$ free parameters for a solution $f$ of $(3.1)$, showing that the kernel of $\mathcal{D}^{s}$ has dimension $s$. In the case $s=1$ this means that the only solution of $\mathcal{D} f=0$ is given by $f(x, \theta)=c$ for some $c \in \mathbb{C}$. Thus, the "parasupercovariant constant function" (meaning that $\mathcal{D} f=0$ ) is indeed given by a constant value $f(x, \theta)=c$. In the case $s=2$ the "Laplace equation" $\mathcal{D}^{2} f=0$ in one dimension has two linearly independent solutions. Consider the inhomogeneous version of (3.1), i.e.,

$$
\mathcal{D}^{s} f(x, \theta)=g(x, \theta) \text {. }
$$

A particular solution is given by $\mathcal{D}^{-s} g$. For any solution $f_{\text {hom }}$ of the homogeneous equation the sum $f_{\text {hom }}+\mathcal{D}^{-s} g$ is a solution of the inhomogeneous equation. Thus, the set of solutions is an affine space (as in the usual "bosonic" case). Let us summarize these observations in the following proposition.

Proposition 3.1. Let the differential equation (3.1) with $s=s^{\prime}(p+1)+s^{\prime \prime}$ where $0 \leq s^{\prime \prime}<p+1$ be given. Any solution of (3.1) can be written as

$$
f_{\text {hom }}(x, \theta)=\sum_{k=0}^{s^{\prime \prime}-1}\left\{\sum_{r=0}^{s^{\prime}} c_{k, r} x^{r}\right\} \theta^{k}+\sum_{k=s^{\prime \prime}}^{p}\left\{\sum_{r=0}^{s^{\prime}-1} c_{k, r} x^{r}\right\} \theta^{k},
$$

where $c_{k, r} \in \mathbb{C}$. Thus, the space of solutions is a complex linear space of dimension s. The associated inhomogeneous differential equation (3.2) has the particular solution $\mathcal{D}^{-s} g$. For any solution $f_{\text {hom }}$ of the homogeneous equation the sum $f_{\text {hom }}+\mathcal{D}^{-s} g$ is a solution of the inhomogeneous equation. Thus, the set of solutions of (3.2) is a complex affine space of dimension s.

Now, let us turn to the "eigenvalue problem"

$$
\mathcal{D} f(x, \theta)=\lambda f(x, \theta)
$$

for $\lambda \in \mathbb{C}$. From above we know that $f(x, \theta)=C e_{q}\left(\lambda^{p+1} x ; \lambda \theta\right)$ is a solution. To find out whether there exists another solution, we write $f(x, \theta)=\sum_{k=0}^{p} f_{k}(x) \theta^{k}$. Inserting this into (3.3) gives for $k=0, \ldots, p-1$ the recursion relation $f_{k+1}(x)=\frac{\lambda}{[k+1]_{q}} f_{k}(x)$ as well as $\partial_{x} f_{0}(x)=\lambda[p]_{q} ! f_{p}(x)$. Using the recursion relation repeatedly yields $f_{p}=\frac{\lambda^{p}}{\left[p p_{q} !\right.} f_{0}$. Using now the second equation yields the differential equation $\partial_{x} f_{0}(x)=\lambda^{p+1} f_{0}(x)$ for $f_{0}$ with the solution $f_{0}(x)=C e^{\lambda^{p+1}} x$ where $C \in \mathbb{C}$ is a constant. From the recursion relation it follows that $f_{k}(x)=\frac{\lambda^{k}}{[k]_{q} !} C e^{\lambda^{p+1} x}$ and, consequently, that $f(x, \theta)=C e_{q}\left(\lambda^{p+1} x ; \lambda \theta\right)$ is indeed the general solution of the differential equation (3.3). Using (2.6), it is straightforward to consider the slightly more difficult eigenvalue problem

$$
\mathcal{D}^{s} f(x, \theta)=\lambda f(x, \theta), \quad s \in \mathbf{N} .
$$

Namely, let $\lambda^{\frac{1}{s}}$ be one of the $s$ roots satisfying $\left(\lambda^{\frac{1}{s}}\right)^{s}=\lambda$. All $s$ roots are then given by

$$
\left\{\lambda^{\frac{1}{s}}, \mu \lambda^{\frac{1}{s}}, \ldots, \mu^{s-1} \lambda^{\frac{1}{s}}\right\}
$$

where $\left\{1, \mu, \mu^{2}, \ldots, \mu^{s-1}\right\}$ is the cyclic group of order $s$ (isomorphic to $\mathbf{Z}_{s}$ ) consisting of the $s$ roots of unity. Since each function $e_{q}\left(\left(\mu^{k} \lambda^{\frac{1}{s}}\right)^{p+1} x ;\left(\mu^{k} \lambda^{\frac{1}{s}}\right) \theta\right)$ solves (3.4), the solution of (3.4) is given by $f(x, \theta)=\sum_{k=0}^{s-1} C_{k} e_{q}\left(\left(\mu^{k} \lambda^{\frac{1}{s}}\right)^{p+1} x ;\left(\mu^{k} \lambda^{\frac{1}{s}}\right) \theta\right)$. The space of solutions has dimension $s$, as expected. Let us summarize these observations in the following proposition. 
Proposition 3.2. Let the differential equation (3.4) be given. Let $\lambda^{\frac{1}{s}}$ be one of the $s$ roots satisfying $\left(\lambda^{\frac{1}{s}}\right)^{s}=\lambda$ and let $\left\{1, \mu, \mu^{2}, \ldots, \mu^{s-1}\right\}$ be the cyclic group of order s. Any solution of (3.4) can be written as

$$
f(x, \theta)=\sum_{k=0}^{s-1} C_{k} e_{q}\left(\left(\mu^{k} \lambda^{\frac{1}{s}}\right)^{p+1} x ;\left(\mu^{k} \lambda^{\frac{1}{s}}\right) \theta\right),
$$

where $C_{k} \in \mathbb{C}$. In particular, the set of solutions of (3.4) is a complex linear space of dimension s.

Up to now, we have only considered equations where a simple ansatz using the exponential function (2.7) yielded all solutions. In the following sections more complicated expressions in $\mathcal{D}$ will be discussed. Due to the lack of a general product rule these cases will turn out to be more difficult and it is unclear what a "good" ansatz should be. This will appear already in the case of linear differential equations of second order discussed in the next section.

Remark 3.1. Let us denote the set of parasuperfunctions $f \equiv f(x, \theta)$ by $\mathcal{A}$ (where we assume for ease of presentation that the components $f_{k}$ are "sufficiently nice", e.g., in $C^{\infty}$ ). Clearly, it is a linear space (over $\mathbb{C}$ ) and if we also consider as product of elements $f, g \in \mathcal{A}$ the pointwise product (i.e., $(f g)(x, \theta):=f(x, \theta) g(x, \theta))$ it is even a commutive ring with unit (having nilpotent elements). The parasupercovariant "derivative" $\mathcal{D}: \mathcal{A} \rightarrow \mathcal{A}$ is, however, not a derivation in this ring, i.e., $\mathcal{D}(f g) \neq \mathcal{D}(f) g+f \mathcal{D}(g)$. Thus, the structure $(\mathcal{A}, \cdot, \mathcal{D})$ is not a differential ring as considered, e.g., in [55] and the literature given therein. It is tempting to introduce a new product $*$ such that $(\mathcal{A}, *, \mathcal{D})$ becomes a differential ring, i.e., $\mathcal{D}(f * g)=\mathcal{D}(f) * g+f * \mathcal{D}(g)$. However, these conditions on the new product seem to be rather involved and a natural interpretation is lacking.

\section{The linear differential equation of second order}

In this section we will discuss the general linear differential equation of second order. More precisely, we will show the following theorem:

Theorem 4.1. Let $c_{1}, c_{2} \in \mathbb{C}$; the general solution of

$$
\left(\mathcal{D}^{2}+c_{1} \mathcal{D}+c_{2}\right) f(x, \theta)=0
$$

may be obtained as follows. Define $\lambda_{ \pm}:=-\frac{c_{1}}{2} \pm \frac{1}{2} \sqrt{c_{1}^{2}-4 c_{2}}$. In the non-degenerated case where $\lambda_{+} \neq \lambda_{-}$the general solution of (4.1) is given by

$$
f(x, \theta)=C_{1} e_{q}\left(\lambda_{+}^{p+1} x ; \lambda_{+} \theta\right)+C_{2} e_{q}\left(\lambda_{-}^{p+1} x ; \lambda_{-} \theta\right)
$$

with arbitrary $C_{i} \in \mathbb{C}$. In the degenerated case where $c_{1}^{2}=4 c_{2}$ we abbreviate $\alpha:=-\frac{c_{1}}{2}$ (thus $\left.c_{2}=\alpha^{2}\right)$; the general solution is in this case given by

$$
f(x, \theta)=C_{1} e_{q}\left(\alpha^{p+1} x ; \alpha \theta\right)+C_{2}\left((p+1) \alpha^{p} x e_{q}\left(\alpha^{p+1} x ; \alpha \theta\right)+e^{\alpha^{p+1} x} \sum_{k=1}^{p} \frac{k \alpha^{k-1} \theta^{k}}{[k]_{q} !}\right) .
$$

Note that making an ansatz of exponential form (as we did in the last section) yields in the non-degenerated case both solutions and in the degenerated case the first solution. Before giving a proof of the theorem, we single out the cases of smallest $p$ explicitly. 
Corollary 4.1. Consider the degenerated case $\left(\mathcal{D}^{2}-2 \alpha \mathcal{D}+\alpha^{2}\right) f(x, \theta)=0$. A first solution is always (i.e., for arbitrary $p$ ) given by $f_{1}(x, \theta)=e_{q}\left(\alpha^{p+1} x ; \alpha \theta\right)$. A linearly independent solution is given in the case $p=1$ (i.e., for an "ordinary" Grassmann variable) by

$$
f_{2}(x, \theta)=2 \alpha x e^{\alpha^{2} x}+\left(1+2 \alpha^{2} x\right) e^{\alpha^{2} x} \theta .
$$

In the case $p=2$ a second solution is given by

$$
f_{2}(x, \theta)=3 \alpha^{2} x e^{\alpha^{3} x}+\left(1+3 \alpha^{3} x\right) e^{\alpha^{3} x} \theta+\frac{\alpha}{[2]_{q} !}\left(2+3 \alpha^{3} x\right) e^{\alpha^{3} x} \theta^{2} .
$$

Let us now turn to the proof of the theorem. Instead of just inserting the claimed solutions and see whether they fulfill the differential equation we will give a longer proof and show how these solutions are found.

Prof of Theorem 4.1. Let $f(x, \theta)=f_{0}(x)+f_{1}(x) \theta+\cdots+f_{p}(x) \theta^{p}$ be a solution of (4.1). Inserting this and comparing coefficients of equal power in $\theta$ yields the following system of equations:

$$
\begin{aligned}
& f_{k+2}(x)=-c_{1} \frac{1}{[k+2]_{q}} f_{k+1}(x)-c_{2} \frac{1}{[k+2]_{q}[k+1]_{q}} f_{k}, \quad 0 \leq k \leq p-2, \\
& \partial_{x} f_{0}(x)=-c_{1}[p]_{q} ! f_{p}(x)-c_{2}[p-1]_{q} ! f_{p-1}(x), \\
& \partial_{x} f_{1}(x)=-c_{1} \partial_{x} f_{0}(x)-c_{2}[p]_{q} ! f_{p}(x) .
\end{aligned}
$$

Note that in the case $p=1$ the recursion relations (4.3) are vacuous. Only the differential equations (4.4)-(4.5) remain and can be cast into the form

$$
\left(\begin{array}{l}
\partial_{x} f_{0}(x) \\
\partial_{x} f_{1}(x)
\end{array}\right)=\left(\begin{array}{ll}
-c_{2} & -c_{1} \\
c_{1} c_{2} & \left(c_{1}^{2}-c_{2}\right)
\end{array}\right)\left(\begin{array}{l}
f_{0}(x) \\
f_{1}(x)
\end{array}\right)
$$

Let us return to the case of arbitrary $p$. Here we will first solve the recursion relations until we arrive at a similar system of differential equations for the components $f_{0}(x)$ and $f_{1}(x)$ which can be solved. To solve the recursion relations, we introduce for $k=0, \ldots, p-1$ the vectors and matrices

$$
v_{k}(x):=\left(\begin{array}{c}
f_{k}(x) \\
f_{k+1}(x)
\end{array}\right), \quad \mathbf{T}_{k+1}:=\left(\begin{array}{cc}
0 & 1 \\
-\frac{c_{2}}{[k+2]_{q}[k+1]_{q}} & -\frac{c_{1}}{[k+2]_{q}}
\end{array}\right) .
$$

This enables us to write the recursion relation (4.3) in the compact form $v_{k+1}(x)=\mathbf{T}_{k+1} v_{k}(x)$. Iterating this yields

$$
v_{k+1}(x)=\mathbf{T}_{k+1} \mathbf{T}_{k} \cdots \mathbf{T}_{1} v_{0}(x)=\mathbf{F}_{k+1} v_{0}(x) .
$$

Since $v_{p-1}(x)=\mathbf{F}_{p-1} v_{0}(x)$, this allows us to express $f_{p-1}$ and $f_{p}$ through $f_{0}$ and $f_{1}$ once we have determined $\mathbf{F}_{p-1}$. Inserting this in (4.4) and (4.5) will give a system of ordinary differential equations for $f_{0}, f_{1}$. We will now determine $\mathbf{F}_{k}$ for all $k$ since after having determined $f_{0}$ and $f_{1}$ the remaining components $f_{k}$ are calculated using (4.8). Let us write the matrix $\mathbf{F}_{k}$ explicitly as

$$
\mathbf{F}_{k}=\left(\begin{array}{cc}
\frac{f_{k-1}^{(1)}}{[k]_{q} !} & \frac{f_{k-1}^{(2)}}{[k]_{q} !} \\
\frac{f_{k}^{(1)}}{[k+1]_{q} !} & \frac{f_{k}^{(2)}}{[k+1]_{q} !}
\end{array}\right)
$$

Note that we can then write

$$
f_{k}(x)=\frac{f_{k-1}^{(1)}}{[k]_{q} !} f_{0}(x)+\frac{f_{k-1}^{(2)}}{[k]_{q} !} f_{1}(x) .
$$


The relation $\mathbf{F}_{k+1}=\mathbf{T}_{k+1} \mathbf{F}_{k}$ is equivalent to

$$
f_{k+2}^{(i)}=-c_{1} f_{k+1}^{(i)}-c_{2} f_{k}^{(i)}, \quad i=1,2 .
$$

In general, a solution of a generalized Fibonacci sequence $f_{k+2}=-c_{1} f_{k+1}-c_{2} f_{k}$ is obtained as follows: The generating function $x^{2}+c_{1} x+c_{2}$ has zeroes $\lambda_{ \pm}=-\frac{c_{1}}{2} \pm \frac{1}{2} \sqrt{c_{1}^{2}-4 c_{2}}$, so that by Binet's formula the general term is given by $f_{k}=a_{1} \lambda_{+}^{k}+a_{2} \lambda_{-}^{k}$, where the coefficients $a_{1}, a_{2}$ are determined using the (given) initial values $f_{0}, f_{1}$. Note that we have the following relations for the coefficients $c_{i}$ :

$$
c_{1}=-\left(\lambda_{+}+\lambda_{-}\right), \quad c_{2}=\lambda_{+} \lambda_{-} .
$$

Let us now assume that $\lambda_{+}=\lambda_{-}$, i.e., $c_{1}^{2}=4 c_{2}$. We define $c_{1}=-2 \alpha$, so that $c_{2}=\alpha^{2}$. In this case we obtain (for $k=0, \ldots, p-1$ )

$$
\mathbf{F}_{k}=\left(\begin{array}{cc}
-\frac{(k-1) \alpha^{k}}{[k]_{q} !} & \frac{k \alpha^{k-1}}{[k]_{q} !} \\
-\frac{k \alpha^{k+1}}{[k+1]_{q} !} & \frac{(k+1) \alpha^{k}}{[k+1]_{q} !}
\end{array}\right) .
$$

Recalling (4.8), we may now use this for $k=p-1$ to express $f_{p}, f_{p-1}$ through $f_{0}, f_{1}$. Inserting this into (4.4) and (4.5) gives the differential equation $v_{0}^{\prime}(x)=A v_{0}(x)$, where the matrix $A$ is given by

$$
A=\left(\begin{array}{cc}
-p \alpha^{p+1} & (p+1) \alpha^{p} \\
-(p+1) \alpha^{p+2} & (p+2) \alpha^{p+1}
\end{array}\right) .
$$

A has only one eigenvalue $\lambda=\alpha^{p+1}$ with eigenvector $\left(\begin{array}{ll}1 & \alpha\end{array}\right)^{t}$. Changing to the appropriate basis allows us to write

$$
\frac{d}{d x}\left(\begin{array}{c}
f_{0}(x) \\
f_{1}(x)+\alpha f_{0}(x)
\end{array}\right)=\left(\begin{array}{cc}
\alpha^{p+1} & (p+1) \alpha^{p} \\
0 & \alpha^{p+1}
\end{array}\right)\left(\begin{array}{c}
f_{0}(x) \\
f_{1}(x)+\alpha f_{0}(x)
\end{array}\right),
$$

which implies that there exist $C_{1}, C_{2} \in \mathbb{C}$ such that

$$
\begin{aligned}
& f_{0}(x)=\left(C_{1}+x(p+1) \alpha^{p} C_{2}\right) e^{\alpha^{p+1} x}, \\
& f_{1}(x)=\left(\alpha C_{1}+x(p+1) \alpha^{p+1} C_{2}+C_{2}\right) e^{\alpha^{p+1} x} .
\end{aligned}
$$

On the other hand, by (4.13) we obtain that

$$
f_{k}(x)=\frac{k \alpha^{k}}{[k]_{q} !}\left(\frac{1}{\alpha} f_{1}(x)-f_{0}(x)\right)+\frac{\alpha^{k}}{[k]_{q} !} f_{0}(x)
$$

for $k=2,3, \ldots, p$. Hence,

$$
\begin{aligned}
f(x, \theta) & =f_{0}(x)+f_{1}(x) \theta+\sum_{k=2}^{p} f_{k}(x) \theta^{k}=f_{0}(x) \sum_{k=0}^{p} \frac{(\alpha \theta)^{k}}{[k]_{q} !}+\frac{f_{1}(x)-\alpha f_{0}(x)}{\alpha} \sum_{k=0}^{p} \frac{k(\alpha \theta)^{k}}{[k]_{q} !} \\
& =C_{1} e^{\alpha^{p+1} x} \sum_{k=0}^{p} \frac{(\alpha \theta)^{k}}{[k]_{q} !}+C_{2} e^{\alpha^{p+1} x}\left(\sum_{k=1}^{p} \frac{k \alpha^{k-1} \theta^{k}}{[k]_{q} !}+x(p+1) \alpha^{p} \sum_{k=0}^{p} \frac{(\alpha \theta)^{k}}{[k]_{q} !}\right) \\
& =C_{1} e_{q}\left(\alpha^{p+1} x ; \alpha \theta\right)+C_{2}\left((p+1) \alpha^{p} x e_{q}\left(\alpha^{p+1} x ; \alpha \theta\right)+e^{\alpha^{p+1} x} \sum_{k=1}^{p} \frac{k \alpha^{k-1} \theta^{k}}{[k]_{q} !}\right),
\end{aligned}
$$

which completes the proof of this case. 
We now assume that $\lambda_{+} \neq \lambda_{-}$. Thus, we obtain the two generalized Fibonacci sequences $\left(f_{k}^{(1)}\right)_{k \in \mathbb{N}}$ and $\left(f_{k}^{(2)}\right)_{k \in \mathbb{N}}$ which satisfy the same recursion relation (4.11) but with different initial conditions. More precisely, one has for the first sequence $f_{0}^{(1)}=0$ and $f_{1}^{(1)}=-c_{2}$, while the initial values for the second sequence are given by $f_{0}^{(2)}=1$ and $f_{1}^{(2)}=-c_{1}$. Defining $\gamma:=\sqrt{c_{1}^{2}-4 c_{2}}$, we can write the general terms of these sequences with the help of Binet's formula as

$$
\begin{aligned}
& f_{k}^{(1)}=\frac{c_{2}}{\gamma} \mu_{k} \equiv \frac{c_{2}}{\gamma}\left(\lambda_{-}^{k}-\lambda_{+}^{k}\right), \\
& f_{k}^{(2)}=\frac{1}{2} \nu_{k}+\frac{c_{1}}{2 \gamma} \mu_{k} \equiv \frac{1}{2}\left(1-\frac{c_{1}}{\gamma}\right) \lambda_{+}^{k}+\frac{1}{2}\left(1+\frac{c_{1}}{\gamma}\right) \lambda_{-}^{k},
\end{aligned}
$$

thereby defining implicitly $\mu_{k}, \nu_{k}$. In particular, using this for $k=p-2$ in (4.8) yields

$$
f_{p-1}(x)=\frac{f_{p-2}^{(1)}}{[p-1]_{q} !} f_{0}(x)+\frac{f_{p-2}^{(2)}}{[p-1]_{q} !} f_{1}(x), \quad f_{p}(x)=\frac{f_{p-1}^{(1)}}{[p]_{q} !} f_{0}(x)+\frac{f_{p-1}^{(2)}}{[p]_{q} !} f_{1}(x) .
$$

Combining (4.4), (4.5) and (4.15) gives the differential equation

$$
v_{0}^{\prime}(x)=\left(\begin{array}{c}
\partial_{x} f_{0}(x) \\
\partial_{x} f_{1}(x)
\end{array}\right)=B v_{0}(x)
$$

where the matrix $B$ is given by

$$
B=\left(\begin{array}{ll}
-c_{1} f_{p-1}^{(1)}-c_{2} f_{p-2}^{(1)} & -c_{1} f_{p-1}^{(2)}-c_{2} f_{p-2}^{(2)} \\
\left(c_{1}^{2}-c_{2}\right) f_{p-1}^{(1)}+c_{1} c_{2} f_{p-2}^{(1)} & \left(c_{1}^{2}-c_{2}\right) f_{p-1}^{(2)}+c_{1} c_{2} f_{p-2}^{(2)}
\end{array}\right)
$$

Note that this is the generalization of (4.6) for arbitrary $p$. Using (4.11) as well as (4.14), we obtain that

$$
B=\left(\begin{array}{ll}
\frac{c_{2}}{\gamma} \mu_{p} & \frac{1}{2} \nu_{p}+\frac{c_{1}}{2 \gamma} \mu_{p} \\
\frac{c_{2}}{\gamma} \mu_{p+1} & \frac{1}{2} \nu_{p+1}+\frac{c_{1}}{2 \gamma} \mu_{p+1}
\end{array}\right)=\frac{1}{\gamma}\left(\begin{array}{ll}
c_{2} \mu_{p} & -\mu_{p+1} \\
c_{2} \mu_{p+1} & -\mu_{p+2}
\end{array}\right) .
$$

In order to find the eigenvalues of the matrix $B$ let us first find the characteristic polynomial of $B$ :

$$
\operatorname{det}(B-x I)=x^{2}-\frac{1}{\gamma}\left(c_{2} \mu_{p}-\mu_{p+2}\right) x+\frac{c_{2}}{\gamma^{2}}\left(\mu_{p+1}^{2}-\mu_{p} \mu_{p+2}\right) .
$$

Recalling (4.12) and $\gamma=\lambda_{+}-\lambda_{-}$, we obtain that

$$
\operatorname{det}(B-x I)=x^{2}-\nu_{p+1} x+c_{2}^{p+1}=\left(x-\lambda_{+}^{p+1}\right)\left(x-\lambda_{-}^{p+1}\right) .
$$

Hence, the eigenvalues of the matrix $B$ are $\lambda_{-}^{p+1}$ and $\lambda_{+}^{p+1}$ with the eigenvectors $\left(1 \lambda_{-}\right)^{t}$ and $\left(1 \lambda_{+}\right)^{t}$, respectively. Therefore, there exist constants $C_{i} \in \mathbb{C}, i=1,2$, such that

$$
\left(\begin{array}{l}
f_{0}(x) \\
f_{1}(x)
\end{array}\right)=C_{1} e^{\lambda_{-}^{p+1} x}\left(\begin{array}{l}
1 \\
\lambda_{-}
\end{array}\right)+C_{2} e^{\lambda_{+}^{p+1} x}\left(\begin{array}{l}
1 \\
\lambda_{+}
\end{array}\right) .
$$

In the case $p=1$ we are finished since all components $f_{0}(x)$ and $f_{1}(x)$ have been determined. For arbitrary $p$ we use (4.10) and (4.14) to get that

$$
f_{k}(x)=\frac{\frac{c_{2}}{\gamma} \mu_{k-1}}{[k]_{q} !} f_{0}(x)+\frac{\frac{1}{2} \nu_{k-1}+\frac{c_{1}}{2 \gamma} \mu_{k-1}}{[k]_{q} !} f_{1}(x)=\frac{1}{\gamma[k]_{q} !}\left(c_{2} \mu_{k-1} f_{0}(x)-\mu_{k} f_{1}(x)\right)
$$


for all $k=2,3, \ldots, p$. This in turn yields

$$
\begin{aligned}
f(x, \theta) & =\sum_{k=0}^{p} f_{k}(x) \theta^{k}=f_{0}(x)+f_{1}(x) \theta+\sum_{k=2}^{p} \frac{\theta^{k}}{\gamma[k]_{q} !}\left(c_{2} \mu_{k-1} f_{0}(x)-\mu_{k} f_{1}(x)\right) \\
& =f_{0}(x)+f_{1}(x) \theta+\frac{c_{2} f_{0}(x)}{\gamma} \sum_{k=2}^{p} \frac{\mu_{k-1} \theta^{k}}{[k]_{q} !}-\frac{f_{1}(x)}{\gamma} \sum_{k=2}^{p} \frac{\mu_{k} \theta^{k}}{[k]_{q} !} \\
& =f_{0}(x)+f_{1}(x) \theta+\frac{\lambda_{+} f_{0}(x)-f_{1}(x)}{\gamma} \sum_{k=2}^{p} \frac{\left(\lambda_{-} \theta\right)^{k}}{[k]_{q} !}+\frac{f_{1}(x)-\lambda_{-} f_{0}(x)}{\gamma} \sum_{k=2}^{p} \frac{\left(\lambda_{+} \theta\right)^{k}}{[k]_{q} !} .
\end{aligned}
$$

Using (4.16), we find that

$$
f_{0}(x)+f_{1}(x) \theta=C_{1} e^{\lambda_{-}^{p+1} x}\left(1+\lambda_{-} \theta\right)+C_{2} e^{\lambda_{+}^{p+1} x}\left(1+\lambda_{+} \theta\right)
$$

as well as $\lambda_{+} f_{0}(x)-f_{1}(x)=\gamma C_{1} e^{\lambda_{-}^{p+1} x}$ and $f_{1}(x)-\lambda_{-} f_{0}(x)=\gamma C_{2} e^{\lambda_{+}^{p+1} x}$, implying

$$
\begin{aligned}
f(x, \theta)= & C_{1} e^{\lambda_{-}^{p+1} x}\left(1+\lambda_{-} \theta\right)+C_{2} e^{\lambda_{+}^{p+1} x}\left(1+\lambda_{+} \theta\right) \\
& +C_{1} e^{\lambda_{-}^{p+1}} x \sum_{k=2}^{p} \frac{\left(\lambda_{-} \theta\right)^{k}}{[k]_{q} !}+C_{2} e^{\lambda_{+}^{p+1} x} \sum_{k=2}^{p} \frac{\left(\lambda_{+} \theta\right)^{k}}{[k]_{q} !} \\
= & C_{1} e^{\lambda_{-}^{p+1} x} \sum_{k=0}^{p} \frac{\left(\lambda_{-} \theta\right)^{k}}{[k]_{q} !}+C_{2} e^{\lambda_{+}^{p+1} x} \sum_{k=0}^{p} \frac{\left(\lambda_{+} \theta\right)^{k}}{[k]_{q} !} \\
= & C_{1} e_{q}\left(\lambda_{-}^{p+1} x, \lambda_{-} \theta\right)+C_{2} e_{q}\left(\lambda_{+}^{p+1} x, \lambda_{+} \theta\right),
\end{aligned}
$$

which completes the proof.

\section{The linear differential equation of arbitrary order}

In this section we solve the linear differential equation of order $n$, where we assume for ease of presentation that $n \leq p$ (for $n \geq p+1$ one has to consider (2.6) instead of (2.4)). Thus, we are considering the equation

$$
\left(\mathcal{D}^{n}+c_{1} \mathcal{D}^{n-1}+\cdots+c_{n-1} \mathcal{D}+c_{n}\right) f(x, \theta)=0 .
$$

Observe that the restriction $n \leq p$ means that for $p=1$ we only consider $n \leq 1$ - but this case was already considered in the above sections. Thus, the case $p=1$ doesn't lead to new cases here. At the end of the section we consider briefly what happens when $n>p$ (see Proposition 5.1).

The strategy for the solution of the above differential equation consists of two steps: In the first step we consider the linear differential equation associated to one degenerated eigenvalue and in the second step we assemble these results for the differential equation (5.1). The second step is only of a formal nature and the main work consists in finding the solutions of the degenerated differential equation.

In the following we will need multi-indices. Therefore, let us fix the notation. We write

$$
\mathbf{m}_{s}:=\left(m_{0}, m_{1}, \ldots, m_{s}\right)
$$

where $m_{0} \geq 0$ and $m_{i} \geq 1$ for $i=1,2, \ldots, s$ are natural numbers. We also introduce the length of $\mathbf{m}_{s}$ by

$$
\left|\mathbf{m}_{s}\right|:=\sum_{i=0}^{s} m_{i} .
$$


Lemma 5.1. Let $f(x, \theta)=\sum_{k=0}^{p} f_{k}(x) \theta^{k}$ be a solution of the differential equation

$$
(\mathcal{D}-\lambda)^{n} f(x, \theta)=0, \quad 1 \leq n \leq p .
$$

Then there exist $C_{1}, \ldots, C_{n} \in \mathbb{C}$ such that

$$
f_{k}(x)=e^{\lambda^{p+1} x} \frac{\lambda^{k}}{[k]_{q} !} \sum_{s=0}^{n-1}\left\{\sum_{\left|\mathbf{m}_{s}\right| \leq n-1} C_{n-\left|\mathbf{m}_{s}\right|} \lambda^{s-\left|\mathbf{m}_{s}\right|}\left(\begin{array}{c}
k \\
m_{0}
\end{array}\right) \prod_{i=1}^{s}\left(\begin{array}{c}
p+1 \\
m_{i}
\end{array}\right)\right\} \frac{\left(\lambda^{p} x\right)^{s}}{s !},
$$

where $\mathbf{m}_{s}=\left(m_{0}, m_{1}, \ldots, m_{s}\right)$ with $m_{0} \geq 0$ and $m_{i} \geq 1$ for all $i=1,2, \ldots, s$.

Proof. We proceed the proof by induction on $n \geq 1$. For $n=1$, the lemma gives - due to $s=0$ and, hence, $m_{0}=0$ - that $f_{k}(x)=C_{1} e^{\lambda^{p+1} x} \frac{\lambda^{k}}{[k]_{q} !}$ which agrees with the solution of (3.3). Thus, the lemma holds for $n=1$. Now, assume that the lemma holds for $n$, and let us prove it for $n+1$. Let us write

$$
(\mathcal{D}-\lambda)^{n+1} f(x, \theta)=(\mathcal{D}-\lambda)^{n} g(x, \theta)=0
$$

with $g(x, \theta)=(\mathcal{D}-\lambda) f(x, \theta)$. Using the induction hypothesis (for $g(x, \theta)$ ), we obtain that there exist $C_{1}, \ldots, C_{n} \in \mathbb{C}$ such that

$$
\sum_{k=0}^{p} g_{k}(x) \theta^{k}=e^{\lambda^{p+1} x} \sum_{k=0}^{p} \frac{(\lambda \theta)^{k}}{[k]_{q} !} \sum_{s=0}^{n-1}\left\{\sum_{\left|\mathbf{m}_{s}\right| \leq n-1} C_{n-\left|\mathbf{m}_{s}\right|} \lambda^{s-\left|\mathbf{m}_{s}\right|}\left(\begin{array}{c}
k \\
m_{0}
\end{array}\right) \prod_{i=1}^{s}\left(\begin{array}{c}
p+1 \\
m_{i}
\end{array}\right)\right\} \frac{\left(\lambda^{p} x\right)^{s}}{s !} .
$$

On the other hand, recalling $g(x, \theta)=(\mathcal{D}-\lambda) f(x, \theta)$ and using $(2.4)$, we obtain

$$
\sum_{k=0}^{p} g_{k}(x) \theta^{k}=\frac{1}{[p]_{q} !} \partial_{x} f_{0}(x) \theta^{p}+\sum_{k=1}^{p} \frac{[k]_{q} !}{[k-1]_{q} !} f_{k}(x) \theta^{k-1}-\lambda \sum_{k=0}^{p} f_{k}(x) \theta^{k} .
$$

By comparing the coefficients of $\theta^{k}$ of the above two equations, we obtain that

$$
\begin{aligned}
g_{p}(x) & =\frac{1}{[p]_{q} !} \partial_{x} f_{0}(x)-\lambda f_{p}(x), \\
g_{k}(x) & =[k+1]_{q} f_{k+1}(x)-\lambda f_{k}(x), \quad 1 \leq k \leq p-1 .
\end{aligned}
$$

Therefore,

$$
\begin{aligned}
\partial_{x} f_{0}(x)-\lambda^{p+1} f_{0}(x) & =\partial_{x} f_{0}(x)+\lambda \sum_{i=0}^{p-1} \lambda^{i}[p-i]_{q} ! f_{p-i}(x)-\lambda \sum_{i=0}^{p} \lambda^{i}[p-i]_{q} ! f_{p-i}(x) \\
& =\sum_{i=0}^{p} \lambda^{i}[p-i]_{q} ! g_{p-i}(x) .
\end{aligned}
$$

Noting $\partial_{x}\left(e^{-\lambda^{p+1} x} f_{0}(x)\right)=e^{-\lambda^{p+1} x}\left(\partial_{x} f_{0}(x)-\lambda^{p+1} f_{0}(x)\right)$, we conclude that

$$
\partial_{x}\left(e^{-\lambda^{p+1} x} f_{0}(x)\right)=e^{-\lambda^{p+1} x} \sum_{i=0}^{p} \lambda^{i}[p-i]_{q} ! g_{p-i}(x) .
$$

Inserting the expression for $g_{p-i}(x)$ given above, this implies

$$
e^{\lambda^{p+1} x} \partial_{x}\left(e^{-\lambda^{p+1} x} f_{0}(x)\right)
$$




$$
\begin{aligned}
& =\sum_{i=0}^{p} \lambda^{i}[p-i]_{q} ! e^{\lambda^{p+1} x} \frac{\lambda^{p-i}}{[p-i]_{q} !} \sum_{s=0}^{n-1}\left\{\sum_{\left|\mathbf{m}_{s}\right| \leq n-1} C_{n-\left|\mathbf{m}_{s}\right|} \lambda^{s-\left|\mathbf{m}_{s}\right|}\left(\begin{array}{c}
p-i \\
m_{0}
\end{array}\right) \prod_{j=1}^{s}\left(\begin{array}{c}
p+1 \\
m_{j}
\end{array}\right)\right\} \frac{\left(\lambda^{p} x\right)^{s}}{s !} \\
& =e^{\lambda^{p+1} x} \lambda^{p} \sum_{i=0}^{p} \sum_{s=0}^{n-1}\left\{\sum_{\left|\mathbf{m}_{s}\right| \leq n-1} C_{n-\left|\mathbf{m}_{s}\right|} \lambda^{s-\left|\mathbf{m}_{s}\right|}\left(\begin{array}{c}
p-i \\
m_{0}
\end{array}\right) \prod_{j=1}^{s}\left(\begin{array}{c}
p+1 \\
m_{j}
\end{array}\right)\right\} \frac{\left(\lambda^{p} x\right)^{s}}{s !} .
\end{aligned}
$$

Thus,

$$
\partial_{x}\left(e^{-\lambda^{p+1} x} f_{0}(x)\right)=\lambda^{p} \sum_{i=0}^{p} \sum_{s=0}^{n-1}\left\{\sum_{\left|\mathbf{m}_{s}\right| \leq n-1} C_{n-\left|\mathbf{m}_{s}\right|} \lambda^{s-\left|\mathbf{m}_{s}\right|}\left(\begin{array}{c}
p-i \\
m_{0}
\end{array}\right) \prod_{j=1}^{s}\left(\begin{array}{c}
p+1 \\
m_{j}
\end{array}\right)\right\} \frac{\left(\lambda^{p} x\right)^{s}}{s !} .
$$

Using

$$
\frac{\left(\lambda^{p} x\right)^{s}}{s !}=\frac{1}{\lambda^{p}} \partial_{x}\left(\frac{\left(\lambda^{p} x\right)^{s+1}}{(s+1) !}\right),
$$

we have found

$$
\partial_{x}\left(e^{-\lambda^{p+1} x} f_{0}(x)\right)=\partial_{x} \sum_{i=0}^{p} \sum_{s=0}^{n-1}\left\{\sum_{\left|\mathbf{m}_{s}\right| \leq n-1} C_{n-\left|\mathbf{m}_{s}\right|} \lambda^{s-\left|\mathbf{m}_{s}\right|}\left(\begin{array}{c}
p-i \\
m_{0}
\end{array}\right) \prod_{j=1}^{s}\left(\begin{array}{c}
p+1 \\
m_{j}
\end{array}\right)\right\} \frac{\left(\lambda^{p} x\right)^{s+1}}{(s+1) !}
$$

or,

$$
e^{-\lambda^{p+1} x} f_{0}(x)=b+\sum_{s=0}^{n-1}\left\{\sum_{i=0}^{p} \sum_{\left|\mathbf{m}_{s}\right| \leq n-1} C_{n-\left|\mathbf{m}_{s}\right|} \lambda^{s-\left|\mathbf{m}_{s}\right|}\left(\begin{array}{c}
p-i \\
m_{0}
\end{array}\right) \prod_{j=1}^{s}\left(\begin{array}{c}
p+1 \\
m_{j}
\end{array}\right)\right\} \frac{\left(\lambda^{p} x\right)^{s+1}}{(s+1) !}
$$

for some constant $b \in \mathbb{C}$. Thus, using $\sum_{i=0}^{p-m_{0}}\left(\begin{array}{c}p-i \\ m_{0}\end{array}\right)=\left(\begin{array}{c}p+1 \\ m_{0}+1\end{array}\right)$, we find

$$
e^{-\lambda^{p+1} x} f_{0}(x)=b+\sum_{s=1}^{n}\left\{\sum_{\left|\mathbf{m}_{s-1}\right| \leq n-1} C_{n-\left|\mathbf{m}_{s-1}\right|} \lambda^{s-\left(\left|\mathbf{m}_{s-1}\right|+1\right)}\left(\begin{array}{c}
p+1 \\
m_{0}+1
\end{array}\right) \prod_{j=1}^{s-1}\left(\begin{array}{c}
p+1 \\
m_{j}
\end{array}\right)\right\} \frac{\left(\lambda^{p} x\right)^{s}}{s !} .
$$

Introducing a new multi-index $\mathbf{n}_{s}=\left(n_{0}, n_{1}, \ldots, n_{s}\right)$ by setting

$$
n_{0}:=0, \quad n_{1}:=m_{0}+1, \quad n_{i}:=m_{i-1}, \quad 2 \leq i \leq s,
$$

we find $\left|\mathbf{m}_{s-1}\right|=\left|\mathbf{n}_{s}\right|-1$ and, consequently,

$$
e^{-\lambda^{p+1} x} f_{0}(x)=b+\sum_{s=1}^{n}\left\{\sum_{\left|\mathbf{n}_{s}\right| \leq n ; n_{0}=0} C_{n-\left|\mathbf{n}_{s}\right|+1} \lambda^{s-\left|\mathbf{n}_{s}\right|} \prod_{j=1}^{s}\left(\begin{array}{c}
p+1 \\
n_{j}
\end{array}\right)\right\} \frac{\left(\lambda^{p} x\right)^{s}}{s !} .
$$

It is clear that we can consider the constant $b$ as a summand corresponding to $s=0$. Switching in notation back from $\mathbf{n}_{s}$ to $\mathbf{m}_{s}$, this yields finally the result

$$
f_{0}(x)=e^{\lambda^{p+1} x} \sum_{s=0}^{n}\left\{\sum_{\left|\mathbf{m}_{s}\right| \leq n ; m_{0}=0} C_{n+1-\left|\mathbf{m}_{s}\right|} \lambda^{s-\left|\mathbf{m}_{s}\right|} \prod_{j=1}^{s}\left(\begin{array}{c}
p+1 \\
m_{j}
\end{array}\right)\right\} \frac{\left(\lambda^{p} x\right)^{s}}{s !} .
$$

This is indeed the form asserted in (5.2) for $f_{0}(x)$ (note that in the formula there one has - due to $k=0$ - that $m_{0}=0$ holds true). 
Now, let us find $f_{k}(x)$ with $k \geq 1$. By an induction on $k$ using (5.3) we get that

$$
f_{k}(x)=\frac{\lambda^{k}}{[k]_{q} !} f_{0}(x)+\sum_{i=0}^{k-1} \frac{[i]_{q} ! \lambda^{k-1-i}}{[k]_{q} !} g_{i}(x)
$$

Substituting the expression of $g_{i}(x)$ shows that

$$
\begin{aligned}
e^{-\lambda^{p+1} x} f_{k}(x)= & \frac{\lambda^{k}}{[k]_{q} !} \sum_{s=0}^{n}\left\{\sum_{\left|\mathbf{m}_{s}\right| \leq n ; m_{0}=0} C_{n+1-\left|\mathbf{m}_{s}\right|} \lambda^{s-\left|\mathbf{m}_{s}\right|} \prod_{j=1}^{s}\left(\begin{array}{c}
p+1 \\
m_{j}
\end{array}\right)\right\} \frac{\left(\lambda^{p} x\right)^{s}}{s !} \\
& +\sum_{i=0}^{k-1} \frac{\lambda^{k-1}}{[k]_{q} !} \sum_{s=0}^{n-1}\left\{\sum_{\left|\mathbf{m}_{s}\right| \leq n-1} C_{n-\left|\mathbf{m}_{s}\right|} \lambda^{s-\left|\mathbf{m}_{s}\right|}\left(\begin{array}{c}
i \\
m_{0}
\end{array}\right) \prod_{j=1}^{s}\left(\begin{array}{c}
p+1 \\
m_{j}
\end{array}\right)\right\} \frac{\left(\lambda^{p} x\right)^{s}}{s !} \\
= & \frac{\lambda^{k}}{[k]_{q} !} \sum_{s=0}^{n}\left\{\sum_{\left|\mathbf{m}_{s}\right| \leq n ; m_{0}=0} C_{n+1-\left|\mathbf{m}_{s}\right|} \lambda^{s-\left|\mathbf{m}_{s}\right|}\left(\begin{array}{c}
k \\
m_{0}
\end{array}\right) \prod_{j=1}^{s}\left(\begin{array}{c}
p+1 \\
m_{j}
\end{array}\right)\right\} \frac{\left(\lambda^{p} x\right)^{s}}{s !} \\
& +\frac{\lambda^{k}}{[k]_{q} !} \sum_{s=0}^{n-1}\left\{\sum_{\left|\mathbf{m}_{s}\right| \leq n-1} C_{n-\left|\mathbf{m}_{s}\right|} \lambda^{s-\left|\mathbf{m}_{s}\right|-1}\left(\begin{array}{c}
k \\
m_{0}+1
\end{array}\right) \prod_{j=1}^{s}\left(\begin{array}{c}
p+1 \\
m_{j}
\end{array}\right)\right\} \frac{\left(\lambda^{p} x\right)^{s}}{s !} \\
= & \frac{\lambda^{k}}{[k]_{q} !} \sum_{s=0}^{n}\left\{\sum_{\left|\mathbf{m}_{s}\right| \leq n ; m_{0}=0} C_{n+1-\left|\mathbf{m}_{s}\right|} \lambda^{s-\left|\mathbf{m}_{s}\right|}\left(\begin{array}{c}
k \\
m_{0}
\end{array}\right) \prod_{j=1}^{s}\left(\begin{array}{c}
p+1 \\
m_{j}
\end{array}\right)\right\} \frac{\left(\lambda^{p} x\right)^{s}}{s !} \\
& +\frac{\lambda^{k}}{[k]_{q} !} \sum_{s=0}^{n-1}\left\{\sum_{\left|\mathbf{m}_{s}\right| \leq n ; m_{0}>0} C_{n+1-\left|\mathbf{m}_{s}\right|} \lambda^{s-\left|\mathbf{m}_{s}\right|}\left(\begin{array}{c}
k \\
m_{0}
\end{array}\right) \prod_{j=1}^{s}\left(\begin{array}{c}
p+1 \\
m_{j}
\end{array}\right)\right\} \frac{\left(\lambda^{p} x\right)^{s}}{s !} .
\end{aligned}
$$

It follows that

$$
f_{k}(x)=e^{\lambda^{p+1} x} \frac{\lambda^{k}}{[k]_{q} !} \sum_{s=0}^{n}\left\{\sum_{\left|\mathbf{m}_{s}\right| \leq n} C_{n+1-\left|\mathbf{m}_{s}\right|} \lambda^{s-\left|\mathbf{m}_{s}\right|}\left(\begin{array}{c}
k \\
m_{0}
\end{array}\right) \prod_{j=1}^{s}\left(\begin{array}{c}
p+1 \\
m_{j}
\end{array}\right)\right\} \frac{\left(\lambda^{p} x\right)^{s}}{s !},
$$

which completes the proof.

Using the expression given in (5.2) for $f_{k}(x)$ (see Lemma 5.1) together with $f(x, \theta)=$ $\sum_{k=0}^{p} f_{k}(x) \theta^{k}$, we obtain the following result.

Theorem 5.1. Let $f(x, \theta)=\sum_{k=0}^{p} f_{k}(x) \theta^{k}$ be a solution of the differential equation

$$
(\mathcal{D}-\lambda)^{n} f(x, \theta)=0, \quad 1 \leq n \leq p .
$$

Then there exist $C_{1}, \ldots, C_{n} \in \mathbb{C}$ such that

$$
f(x, \theta)=e^{\lambda^{p+1} x} \sum_{k=0}^{p}\left\{\sum_{s=0}^{n-1}\left(\sum_{\left|\mathbf{m}_{s}\right| \leq n-1} C_{n-\left|\mathbf{m}_{s}\right|} \lambda^{s-\left|\mathbf{m}_{s}\right|}\left(\begin{array}{c}
k \\
m_{0}
\end{array}\right) \prod_{i=1}^{s}\left(\begin{array}{c}
p+1 \\
m_{i}
\end{array}\right)\right) \frac{\left(\lambda^{p} x\right)^{s}}{s !}\right\} \frac{(\lambda \theta)^{k}}{[k]_{q} !},
$$

where $\mathbf{m}_{s}=\left(m_{0}, m_{1}, \ldots, m_{s}\right)$ with $m_{0} \geq 0$ and $m_{i} \geq 1$, for all $i=1,2, \ldots, s$.

Example 5.1. Let us consider the case $n=2$ (the case $n=1$ was already considered above during the induction). The formula given in Theorem 5.1 yields for $s$ the two summands corresponding to $s=0$ and $s=1$. Let us consider first $s=0$. It follows that $\left|\mathbf{m}_{0}\right| \leq 1$ and, therefore, that $m_{0}=0$ or $m_{0}=1$. The entire inner sum yields thus $C_{2}+C_{1} \lambda^{-1} k$. In the case $s=1$ the 
only possibility is $\mathbf{m}_{1}=\left(m_{0}, m_{1}\right)=(0,1)$ with $\left|\mathbf{m}_{1}\right|=1$. The inner sum yields in this case $C_{1}(p+1) \lambda^{p} x$. Thus,

$$
\begin{aligned}
f(x, \theta) & =e^{\lambda^{p+1} x} \sum_{k=0}^{p}\left\{C_{2}+C_{1} \lambda^{-1} k+C_{1}(p+1) \lambda^{p} x\right\} \frac{(\lambda \theta)^{k}}{[k]_{q} !} \\
& =C_{2} e^{\lambda^{p+1}} x \sum_{k=0}^{p} \frac{(\lambda \theta)^{k}}{[k]_{q} !}+C_{1}\left((p+1) \lambda^{p} x e^{\lambda^{p+1} x} \sum_{k=0}^{p} \frac{(\lambda \theta)^{k}}{[k]_{q} !}+e^{\lambda^{p+1} x} \sum_{k=0}^{p} \frac{k \lambda^{k-1} \theta^{k}}{[k]_{q} !}\right) \\
& =C_{2} e_{q}\left(\lambda^{p+1} x ; \lambda \theta\right)+C_{1}\left((p+1) \lambda^{p} x e_{q}\left(\lambda^{p+1} x ; \lambda \theta\right)+e^{\lambda^{p+1} x} \sum_{k=1}^{p} \frac{k \lambda^{k-1} \theta^{k}}{[k]_{q} !}\right) .
\end{aligned}
$$

This is exactly equation (4.2) given in Theorem 4.1 for the case $n=2$ (with degenerated eigenvalue).

Using the linearity of the general case, equation (5.1) and Theorem 5.1 we get the following theorem.

Theorem 5.2. Let the differential equation (5.1) with $1 \leq n \leq p$ be given. Assume that the different roots $\lambda_{1}, \ldots, \lambda_{m}$ of the characteristic polynomial $x^{n}+c_{1} x^{n-1}+\cdots+c_{n-1} x+c_{n}$ have multiplicities $n_{1}, \ldots, n_{m}$ (with $n_{1}+\cdots+n_{m}=n$ and $n_{i} \geq 1$ ). Then the solution $f(x, \theta)$ of the differential equation (5.1) can be written as

$$
f(x, \theta)=\sum_{i=1}^{m} e^{\lambda_{i}^{p+1} x} \sum_{k=0}^{p}\left\{\sum_{s=0}^{n_{i}-1}\left(\sum_{\left|\mathbf{m}_{s}\right| \leq n_{i}-1} C_{n_{i}-\left|\mathbf{m}_{s}\right|}^{(i)} \lambda_{i}^{s-\left|\mathbf{m}_{s}\right|}\left(\begin{array}{c}
k \\
m_{0}
\end{array}\right) \prod_{i=1}^{s}\left(\begin{array}{c}
p+1 \\
m_{i}
\end{array}\right)\right) \frac{\left(\lambda_{i}^{p} x\right)^{s}}{s !}\right\} \frac{\left(\lambda_{i} \theta\right)^{k}}{[k]_{q} !}
$$

where $C_{1}^{(i)}, \ldots, C_{n_{i}}^{(i)} \in \mathbb{C}$ are some constants (there are $n$ of them) and $\mathbf{m}_{s}=\left(m_{0}, m_{1}, \ldots, m_{s}\right)$ with $m_{0} \geq 0$ and $m_{i} \geq 1$, for all $i=1,2, \ldots, s$.

This theorem immediately implies the following corollary.

Corollary 5.1. Let the differential equation (5.1) with $1 \leq n \leq p$ be given. The set of its solutions is a complex linear space of dimension $n$. The set of solutions of the corresponding inhomogeneous differential equation

$$
\left(\mathcal{D}^{n}+c_{1} \mathcal{D}^{n-1}+\cdots+c_{n-1} \mathcal{D}+c_{n}\right) f(x, \theta)=g(x, \theta)
$$

is an $n$-dimensional complex affine space.

Proof. It only remains to check the last assertion. The argument is the same as in the usual case: If $f_{i}(x, \theta)$ is a particular solution of the inhomogeneous differential equation then any function of the form $f_{\text {hom }}(x, \theta)+f_{i}(x, \theta)$ - where $f_{\text {hom }}(x, \theta)$ is a solution of the homogeneous differential equation - satisfies the inhomogeneous differential equation.

Remark 5.1. An approach similar to the case $n=2$ is also possible in the more general case where $2 \leq n \leq p$. Inserting $f(x, \theta)=f_{0}(x)+\cdots+f_{p}(x) \theta^{p}$ into (5.1) yields after a straightforward, but slightly tedious computation in close analogy to (4.3)-(4.5) the following system

$$
\begin{aligned}
& f_{k+n}(x)=-\sum_{i=1}^{n} c_{i} \frac{[k+n-i]_{q} !}{[k+n]_{q} !} f_{k+n-i}(x), \quad 0 \leq k \leq p-n, \\
& \partial_{x} f_{k+n-p-1}(x)=-\sum_{i=1}^{k+n-p-1} c_{i} \frac{[k+n-p-1-i]_{q} !}{[k+n-p-1]_{q} !} \partial_{x} f_{k+n-p-1-i}(x)
\end{aligned}
$$




$$
-\sum_{i=k+n-p}^{n} c_{i} \frac{[k+n-i]_{q} !}{[k+n-p-1]_{q} !} f_{k+n-i}(x), \quad p-n+1 \leq k \leq p .
$$

Note that this system constitutes indeed $p+1$ conditions as it should and that this system reduces for $n=2$ to the one given in (4.3)-(4.5) (here the recursion relations (5.4) are also vacuous in the case $p=1$ and only the system of differential equations remains - as in the case $n=2)$. Considering the recursion relation and abbreviating $\gamma_{i}(k):=-c_{i} \frac{[k+n-i]_{q} !}{[k+n]_{q} !}$, we may write (5.4) as

$$
\left(\begin{array}{c}
f_{k+1}(x) \\
\vdots \\
f_{k+n}(x)
\end{array}\right)=\left(\begin{array}{cccccc}
0 & 1 & 0 & \cdots & \cdots & 0 \\
\vdots & \ddots & 1 & \ddots & & \vdots \\
\vdots & & \ddots & \ddots & \ddots & \vdots \\
\vdots & & & \ddots & 1 & 0 \\
0 & \cdots & \cdots & \cdots & 0 & 1 \\
\gamma_{n}(k) & \gamma_{n-1}(k) & \cdots & & \cdots & \gamma_{1}(k)
\end{array}\right)\left(\begin{array}{c}
f_{k}(x) \\
\vdots \\
f_{k+n-1}(x)
\end{array}\right)
$$

Let us denote the above $n \times n$ matrix by $\mathbf{T}_{k+1}^{(n)}$ and the vector on the right hand side by $v_{k}(x)$; observe that $\mathbf{T}_{k+1}^{(2)}$ equals $\mathbf{T}_{k+1}$ from (4.7). We may, therefore, write the above equation as $v_{k+1}(x)=\mathbf{T}_{k+1}^{(n)} v_{k}(x)$. This implies that $v_{p-n}(x)=\mathbf{T}_{p-n}^{(n)} \cdots \mathbf{T}_{1}^{(n)} v_{0}(x)$. Let $\mathbf{F}_{k+1}^{(n)}:=$ $\mathbf{T}_{k+1}^{(n)} \cdots \mathbf{T}_{1}^{(n)}$. As in the case $n=2$ considered in the last section, we may reformulate the recursion $\mathbf{F}_{k+1}^{(n)}=\mathbf{T}_{k+1}^{(n)} \mathbf{F}_{k}^{(n)}$ by considering appropriately normalized entries of the matrices $\mathbf{F}_{k}^{(n)}$. More precisely, we introduce in analogy to (4.9) the entries $f_{m}^{(j)}$ by

$$
\mathbf{F}_{k}^{(n)}=\left(\frac{f_{k-n+i}^{(j)}}{[k-n+i+1]_{q} !}\right)_{i, j=1, \ldots, n} .
$$

This implies for each $j$ with $j=1, \ldots, n$ the recursion relation

$$
f_{k+n}^{(j)}=-c_{1} f_{k+n-1}^{(j)}-c_{2} f_{k+n-2}^{(j)}-\cdots-c_{n} f_{k}^{(j)} .
$$

Thus, for each $j$ there will be an $n$-generalized Fibonacci sequence $\left(f_{k}^{(j)}\right)_{k \in \mathbb{N}}$ satisfying (5.6) [56, 57]; they are differing only in the initial values $\left(f_{0}^{(j)}, \ldots, f_{n-1}^{(j)}\right)$. The initial values of $\left(f_{k}^{(j)}\right)_{k \in \mathbb{N}}$, are exactly the normalized entries in the $j$-th column of $\mathbf{T}_{1}^{(n)}$. This is the generalization of (4.11). To proceed further in close analogy to the case $n=2$ one denotes the zeroes of the generating function $x^{n}+c_{1} x^{n-1}+\cdots+c_{n}$ by $\lambda_{1}, \ldots, \lambda_{n}$, where some of these zeroes may occur with multiplicity greater than one. By using the appropriate generalization of Binet's formula (cf. [56, $57,58]$ ) one can write the general solution as $f_{k}=a_{1} \lambda_{1}^{k}+\cdots+a_{n} \lambda_{n}^{k}$, where the $a_{i}$ are determined by the initial values. However, the resulting expressions become rather unpleasant so that we won't consider this procedure further. It is, however, clear that this will lead in the second step after inserting the resulting relations into (5.5) - to a system of linear differential equations which has then to be solved.

In the above considerations concerning (5.1) we have always assumed that $2 \leq n \leq p$. Recall that the motivation for the restriction $n \leq p$ comes from the periodicity (2.5) which shows that in this case derivatives of at most first order in the components $f_{k}(x)$ appear, see (2.4), whereas in the case $n \geq p+1$ derivatives of higher order appear, see (2.6). In the remaining part of the section we consider the case where an arbitrary $n$ is allowed. It is to be expected that (5.1) is equivalent to a system of ordinary differential equations of higher order. 
Proposition 5.1. Let the differential equation (5.1) be given where $n=n^{\prime}(p+1)+n^{\prime \prime}$ with $0 \leq n^{\prime \prime}<p+1$. This differential equation for $f(x, \theta)=f_{0}(x)+f_{1}(x) \theta+\cdots+f_{p}(x) \theta^{p}$ is equivalent to the following system of differential equations for the components $f_{k}(x)$

$$
\begin{aligned}
& \sum_{\nu=0}^{n^{\prime \prime}} c_{n^{\prime \prime}-\nu}[\nu]_{q} ! \partial_{x}^{n^{\prime}} f_{\nu}(x)=-\sum_{\mu=0}^{n^{\prime}-1} \sum_{\nu=0}^{p} c_{\left(n^{\prime}-\mu\right)(p+1)+\left(n^{\prime \prime}-\nu\right)}[\nu]_{q} ! \partial_{x}^{\mu} f_{\nu}(x), \\
& \sum_{\nu=0}^{n^{\prime \prime}} c_{n^{\prime \prime}-\nu}[l+\nu]_{q} ! \partial_{x}^{n^{\prime}} f_{l+\nu}(x)=-\sum_{\mu=0}^{n^{\prime}-1}\left(\sum_{\nu=0}^{p-l} c_{\left(n^{\prime}-\mu\right)(p+1)+\left(n^{\prime \prime}-\nu\right)}[l+\nu]_{q} ! \partial_{x}^{\mu} f_{l+\nu}(x)\right. \\
& \left.+\sum_{\nu=p-l+1}^{p} c_{\left(n^{\prime}-\mu\right)(p+1)+\left(n^{\prime \prime}-\nu\right)}[\underline{l+\nu}]_{q} ! \partial_{x}^{\mu} \underline{f_{l+\nu}}(x)\right), \\
& \sum_{\nu=p+1-l}^{n^{\prime \prime}} c_{n^{\prime \prime}-\nu}[l+\nu]_{q} ! \partial_{x}^{n^{\prime}+1} f_{\underline{l+\nu}}(x)=-\sum_{\nu=0}^{p-l} c_{n^{\prime \prime}-\nu}[l+\nu]_{q} ! \partial_{x}^{n^{\prime}} f_{l+\nu}(x) \\
& -\sum_{\mu=0}^{n^{\prime}-1}\left(\sum_{\nu=0}^{p-l} c_{\left(n^{\prime}-\mu\right)(p+1)+\left(n^{\prime \prime}-\nu\right)}[l+\nu]_{q} ! \partial_{x}^{\mu} f_{l+\nu}(x)\right. \\
& \left.+\sum_{\nu=p-l+1}^{p} c_{\left(n^{\prime}-\mu\right)(p+1)+\left(n^{\prime \prime}-\nu\right)}[\underline{l+\nu}]_{q} ! \partial_{x}^{\mu} f_{\underline{l+\nu}}(x)\right) \text {, }
\end{aligned}
$$

where we have introduced the abbreviation $\underline{x}:=x-(p+1)$ as well as $c_{0}:=1$. The second equation holds for all $l$ with $1 \leq l \leq p-n^{\prime \prime}$ and the third equation for all $l$ with $p-n^{\prime \prime}+1 \leq l \leq p$.

Before turning to the proof we would like to point out a few facts. Note first that there are in total $p+1$ equations which have to be satisfied, as it should be. As a second point note that there are $n^{\prime \prime}$ differential equations of order $n^{\prime}+1$ as well as $p-n^{\prime \prime}+1$ differential equations of order $n^{\prime}$. The case $n \leq p$ considered before corresponds to $n^{\prime}=0$ and $n^{\prime \prime}=n$ : The above proposition yields that there are $n$ differential equations of order 1 as well as $p-n+1$ differential equations of order 0 - which is indeed the case, see (5.4)-(5.5). On the other hand, if $n=n^{\prime}(p+1)$, i.e., $n^{\prime \prime}=0$, then one has $p+1$ differential equations of order $n^{\prime}$ (and none of higher order). Thus, in this case all differential equations have the same order.

Corollary 5.2. Let the differential equation (5.1) be given where $n=n^{\prime}(p+1)+n^{\prime \prime}$ with $0 \leq n^{\prime \prime}<p+1$. Then (5.1) is equivalent to a set of $n^{\prime \prime}$ differential equations of order $n^{\prime}+1$ as well as $p-n^{\prime \prime}+1$ differential equations of order $n^{\prime}$ for the components $f_{k}(x)$. In particular, the set of solutions of (5.1) is a complex linear space of dimension $n$.

Proof. It remains to check the dimension of the space of solutions. However, since the linear differential equation of order $n^{\prime}$ (resp. $n^{\prime}+1$ ) has $n^{\prime}\left(\right.$ resp. $\left.n^{\prime}+1\right)$ linear independent solutions, one has in total $n^{\prime \prime}\left(n^{\prime}+1\right)+\left(p-n^{\prime \prime}+1\right) n^{\prime}=n^{\prime}(p+1)+n^{\prime \prime}=n$ linear independent solutions.

Let us check that the above equations given in Proposition 5.1 do indeed reduce to the ones given in (5.4)-(5.5) for the case $n \leq p$, i.e., $n^{\prime}=0$ and $n^{\prime \prime}=n$. Consider the first equation of Proposition 5.1. Since $n^{\prime}=0$ the right-hand side vanishes. It follows that $c_{0}[n]_{q} ! f_{n}(x)=$ $-\sum_{\nu=0}^{n-1} c_{n-\nu}[\nu]_{q} ! f_{\nu}(x)$. Recalling $c_{0}=1$ and introducing $i=n-\nu$, this is equivalent to

$$
f_{n}(x)=-\sum_{\nu=0}^{n-1} c_{i} \frac{[n-i]_{q} !}{[n]_{q} !} f_{n-i}(x)
$$


which is the case $k=0$ of (5.4). In the second equation of Proposition 5.1 the right-hand side also vanishes, implying $\sum_{\nu=0}^{n} c_{n-\nu}[l+\nu]_{q} ! f_{l+\nu}(x)=0$. Using $c_{0}=1$ and introducing $i=n-\nu$ yields as above

$$
f_{l+n}(x)=-\sum_{i=1}^{n} c_{i} \frac{[l+n-i]_{q} !}{[l+n]_{q} !} f_{l+n-i}(x)
$$

which are the (remaining) cases $1 \leq l \leq p-n$ of (5.4). In the third equation of Proposition 5.1 only the first sum remains, implying

$$
\sum_{\nu=p+1-l}^{n} c_{n-\nu}[l+\nu-(p+1)]_{q} ! \partial_{x} f_{l+\nu-(p+1)}(x)=-\sum_{\nu=0}^{p-l} c_{n-\nu}[l+\nu]_{q} ! f_{l+\nu}(x) .
$$

Singling out again the summand $\nu=n$, recalling $c_{0}=1$ and introducing $i=n-\nu$ yields

$$
\begin{aligned}
{[l+n-(p+1)]_{q} ! \partial_{x} f_{l+n-(p+1)}(x)=} & -\sum_{i=1}^{l+n-(p+1)} c_{i}[l+n-(p+1)-i]_{q} ! \partial_{x} f_{l+n-(p+1)-i}(x) \\
& -\sum_{i=n+l-p}^{n} c_{i}[l+n-i]_{q} ! f_{l+n-i}(x)
\end{aligned}
$$

which is exactly (5.5). Thus, the system of equations given in Proposition 5.1 reduces for $n \leq p$ indeed to (5.4)-(5.5). Now, let us turn to the proof of the proposition.

Proof of Proposition 5.1. The proof is straightforward but slightly tedious since one has to be very careful with the indices involved. Let us introduce $c_{0}:=1$ as well as $d_{s}:=c_{n-s}$ for $0 \leq s \leq n$. Recalling furthermore that we can split $n$ in a unique fashion as $n=n^{\prime}(p+1)+n^{\prime \prime}$ with $0 \leq n^{\prime \prime} \leq p$, we can write the left-hand side of (5.1) in the following form

$$
\sum_{s=0}^{n} d_{s} \mathcal{D}^{s} f(x, \theta)=\sum_{\mu=0}^{n^{\prime}-1} \sum_{\nu=0}^{p} d_{\mu(p+1)+\nu} \mathcal{D}^{\mu(p+1)+\nu} f(x, \theta)+\sum_{\nu=0}^{n^{\prime \prime}} d_{n^{\prime}(p+1)+\nu} \mathcal{D}^{n^{\prime}(p+1)+\nu} f(x, \theta) .
$$

Inserting the expansion $f(x, \theta)=f_{0}(x)+f_{1}(x) \theta+\cdots+f_{p}(x) \theta^{p}$ and using (2.6) yields

$$
\begin{aligned}
\sum_{s=0}^{n} d_{s} \mathcal{D}^{s} f(x, \theta)= & \sum_{\mu=0}^{n^{\prime}-1} \sum_{\nu=0}^{p} \sum_{k=0}^{\nu-1} d_{\mu(p+1)+\nu} \frac{[k]_{q} !}{[p-(\nu-1)+k]_{q} !} \partial_{x}^{\mu+1} f_{k}(x) \theta^{p-(\nu-1)+k} \\
& +\sum_{\mu=0}^{n^{\prime}-1} \sum_{\nu=0}^{p} \sum_{k=\nu}^{p} d_{\mu(p+1)+\nu} \frac{[k]_{q} !}{[k-\nu]_{q} !} \partial_{x}^{\mu} f_{k}(x) \theta^{k-\nu} \\
& +\sum_{\nu=0}^{n^{\prime \prime}} \sum_{k=0}^{\nu-1} d_{n^{\prime}(p+1)+\nu} \frac{[k]_{q} !}{[p-(\nu-1)+k]_{q} !} \partial_{x}^{n^{\prime}+1} f_{k}(x) \theta^{p-(\nu-1)+k} \\
& +\sum_{\nu=0}^{n^{\prime \prime}} \sum_{k=\nu}^{p} d_{n^{\prime}(p+1)+\nu} \frac{[k]_{q} !}{[k-\nu]_{q} !} \partial_{x}^{n^{\prime}} f_{k}(x) \theta^{k-\nu} .
\end{aligned}
$$

We now consider the terms on the right-hand side of this equation separately. Let us begin with the second term. Introducing $l=k-\nu$, we switch from the indices $k, \nu$ to $l, \nu$ and write this term equivalently as

$$
\sum_{l=0}^{p} \sum_{\nu=0}^{p-l} \sum_{\mu=0}^{n^{\prime}-1} d_{\mu(p+1)+\nu} \frac{[l+\nu]_{q} !}{[l]_{q} !} \partial_{x}^{\mu} f_{l+\nu}(x) \theta^{l}
$$


Let us turn to the fourth term. Here we also introduce $l=k-\nu$ and switch from the indices $k, \nu$ to $l, \nu$. Treating the range of $\nu$ carefully, this term can equivalently be written as

$$
\sum_{l=0}^{p} \sum_{\nu=0}^{\min \left(p-l, n^{\prime \prime}\right)} d_{n^{\prime}(p+1)+\nu} \frac{[l+\nu]_{q} !}{[l]_{q} !} \partial_{x}^{n^{\prime}} f_{l+\nu}(x) \theta^{l} .
$$

In the first term we introduce $l=p+1-(\nu-k)$; it ranges from 1 to $p$. Switching from the indices $k, \nu$ to $l, \nu$, this term is equivalent to

$$
\sum_{l=1}^{p} \sum_{\nu=p+1}^{p} \sum_{\mu=0}^{n^{\prime}-1} d_{\mu(p+1)+\nu} \frac{[l+\nu-(p+1)]_{q} !}{[l]_{q} !} \partial_{x}^{\mu} f_{l+\nu-(p+1)}(x) \theta^{l} .
$$

Treating the third term in the same fashion (carefully treating the ranges) yields

$$
\sum_{l=p+1-n^{\prime \prime}}^{p} \sum_{\nu=p+1-l}^{n^{\prime \prime}} d_{n^{\prime}(p+1)+\nu} \frac{[l+\nu-(p+1)]_{q} !}{[l]_{q} !} \partial_{x}^{n^{\prime}+1} f_{l+\nu-(p+1)}(x) \theta^{l} .
$$

Inspecting the resulting expressions for the four terms we see that upon combining them in powers of $\theta$ there are three ranges to consider: 1) The range $l=0$; here one has a contribution from the second and fourth term. 2) The range $1 \leq l \leq p-n^{\prime \prime}$; here one has a contribution from the second and fourth term as well as the first term. 3) The range $p-n^{\prime \prime}+1 \leq l \leq p$; here all terms contribute. We can, therefore, write

$$
\sum_{s=0}^{n} d_{s} \mathcal{D}^{s} f(x, \theta)=E_{0}(x)+\sum_{l=1}^{p-n^{\prime \prime}} F_{l}(x) \theta^{l}+\sum_{l=p+1-n^{\prime \prime}}^{p} G_{l}(x) \theta^{l},
$$

where we have abbreviated the corresponding functions $E_{0}(x), F_{l}(x)$ and $G_{l}(x)$ as follows:

$$
\begin{aligned}
E_{0}(x)= & \sum_{\nu=0}^{p} \sum_{\mu=0}^{n^{\prime}-1} d_{\mu(p+1)+\nu}[\nu]_{q} ! \partial_{x}^{\mu} f_{\nu}(x)+\sum_{\nu=0}^{n^{\prime \prime}} d_{n^{\prime}(p+1)+\nu}[\nu]_{q} ! \partial_{x}^{n^{\prime}} f_{\nu}(x), \\
F_{l}(x)= & \sum_{\nu=0}^{p-l} \sum_{\mu=0}^{n^{\prime}-1} d_{\mu(p+1)+\nu} \frac{[l+\nu]_{q} !}{[l]_{q} !} \partial_{x}^{\mu} f_{l+\nu}(x)+\sum_{\nu=0}^{\min \left(p-l, n^{\prime \prime}\right)} d_{n^{\prime}(p+1)+\nu} \frac{[l+\nu]_{q} !}{[l]_{q} !} \partial_{x}^{n^{\prime}} f_{l+\nu}(x) \\
& +\sum_{\nu=p+1}^{p} \sum_{\mu=0}^{n^{\prime}-1} d_{\mu(p+1)+\nu} \frac{\left[\underline{l+\nu]_{q} !}\right.}{[l]_{q} !} \partial_{x}^{\mu} f_{\underline{l+\nu}}(x), \\
G_{l}(x)= & F_{l}(x)+\sum_{\nu=p+1-l}^{n^{\prime \prime}} d_{n^{\prime}(p+1)+\nu} \frac{[l+\nu]_{q} !}{[l]_{q} !} \partial_{x}^{n^{\prime}+1} f_{\underline{l+\nu}}(x) .
\end{aligned}
$$

Thus, (5.1) is equivalent to $E_{0}(x)=0, F_{l}(x)=0=G_{l}(x)$ for all $l$. Let us start with $E_{0}(x)=0$. This is equivalent to

$$
\sum_{\nu=0}^{n^{\prime \prime}} d_{n^{\prime}(p+1)+\nu}[\nu]_{q} ! \partial_{x}^{n^{\prime}} f_{\nu}(x)=-\sum_{\mu=0}^{n^{\prime}-1} \sum_{\nu=0}^{p} d_{\mu(p+1)+\nu}[\nu]_{q} ! \partial_{x}^{\mu} f_{\nu}(x) .
$$

Recalling $d_{s}=c_{n-s}$ as well as $n=n^{\prime}(p+1)+n^{\prime \prime}$, this is equal to

$$
\sum_{\nu=0}^{n^{\prime \prime}} c_{n^{\prime \prime}-\nu}[\nu]_{q} ! \partial_{x}^{n^{\prime}} f_{\nu}(x)=-\sum_{\mu=0}^{n^{\prime}-1} \sum_{\nu=0}^{p} c_{\left(n^{\prime}-\mu\right)(p+1)+\left(n^{\prime \prime}-\nu\right)}[\nu]_{q} ! \partial_{x}^{\mu} f_{\nu}(x),
$$


which is the first asserted equation. Let us turn to $F_{l}(x)=0$. The highest order of a derivative appearing is $n^{\prime}$. Thus, switching also to the coefficients $c_{s}$, we can write $F_{l}(x)=0$ (with $\left.1 \leq l \leq p-n^{\prime \prime}\right)$ equivalently as

$$
\begin{aligned}
\sum_{\nu=0}^{n^{\prime \prime}} c_{n^{\prime \prime}-\nu}[l+\nu]_{q} ! \partial_{x}^{n^{\prime}} f_{l+\nu}(x)= & -\sum_{\mu=0}^{n^{\prime}-1}\left(\sum_{\nu=0}^{p-l} c_{\left(n^{\prime}-\mu\right)(p+1)+\left(n^{\prime \prime}-\nu\right)}[l+\nu]_{q} ! \partial_{x}^{\mu} f_{l+\nu}(x)\right. \\
& \left.+\sum_{\nu=p-l+1}^{p} c_{\left(n^{\prime}-\mu\right)(p+1)+\left(n^{\prime \prime}-\nu\right)}[\underline{l+\nu}]_{q} ! \partial_{x}^{\mu} f_{\underline{l+\nu}}(x)\right),
\end{aligned}
$$

which is the asserted second equation. It remains to consider $G_{l}(x)=0$. Here derivatives of order $n^{\prime}+1$ appear. Thus, switching also to the coefficients $c_{s}$, we can write $G_{l}(x)=0$ (with $\left.p-n^{\prime \prime}+1 \leq l \leq p\right)$ equivalently as

$$
\begin{aligned}
\sum_{\nu=p+1-l}^{n^{\prime \prime}} c_{n^{\prime \prime}-\nu}[\underline{l+\nu}]_{q} ! \partial_{x}^{n^{\prime}+1} f_{\underline{l+\nu}}(x)= & -\sum_{\nu=0}^{p-l} c_{n^{\prime \prime}-\nu}[l+\nu]_{q} ! \partial_{x}^{n^{\prime}} f_{l+\nu}(x) \\
& -\sum_{\mu=0}^{n^{\prime}-1}\left(\sum_{\nu=0}^{p-l} c_{\left(n^{\prime}-\mu\right)(p+1)+\left(n^{\prime \prime}-\nu\right)}[l+\nu]_{q} ! \partial_{x}^{\mu} f_{l+\nu}(x)\right. \\
& \left.+\sum_{\nu=p-l+1}^{p} c_{\left(n^{\prime}-\mu\right)(p+1)+\left(n^{\prime \prime}-\nu\right)}[l+\nu]_{q} ! \partial_{x}^{\mu} \underline{f l+\nu}_{\underline{l+1}}(x)\right),
\end{aligned}
$$

which is the asserted third equation.

\section{$6 \quad$ Linear systems of first order}

Using the standard procedure to transform a linear differential equation into a system of differential equations of first order, we define $v_{1}(x, \theta):=f(x, \theta)$ as well as $v_{j}(x, \theta):=\mathcal{D}^{j-1} f(x, \theta)$ for $2 \leq j \leq n$. Forming the vector function $v(x, \theta):=\left(v_{1}(x, \theta), \ldots, v_{n}(x, \theta)\right)^{t}$, we can transform the differential equation (5.1) into the form $\mathcal{D} v(x, \theta)=A v(x, \theta)$ where the matrix $A \in \mathbb{C}^{n, n}$ is given in the usual form. Clearly, one is interested in the general system

$$
\mathcal{D} w(x, \theta)=A w(x, \theta),
$$

where the matrix $A \in \mathbb{C}^{n, n}$ is arbitrary and $w(x, \theta)=\left(w_{1}(x, \theta), \ldots, w_{n}(x, \theta)\right)^{t}$ is a function to be determined. What one is in fact looking for is the generalization of the function $e_{q}\left(a^{p+1} x ; a \theta\right) \equiv$ $e^{a^{p+1} x} \sum_{k=0}^{p} \frac{(a \theta)^{k}}{[k]_{q} !}$ satisfying $\mathcal{D} e_{q}\left(a^{p+1} x ; a \theta\right)=a e_{q}\left(a^{p+1} x ; a \theta\right)$ to the case with matrix arguments.

Proposition 6.1. Let $A \in \mathbb{C}^{n, n}$ be given. For a given $p \geq 1$ define the matrix exponential function $E_{q}$ by

$$
E_{q}\left(A^{p+1} x ; A \theta\right):=e^{A^{p+1} x} \sum_{k=0}^{p} \frac{(A \theta)^{k}}{[k]_{q} !} .
$$

The so defined function $E_{q}$ has the following properties:

1. In the scalar case $n=1$ it reduces to the exponential function $e_{q}$.

2. If the matrix $A$ has diagonal form, i.e., $A=\operatorname{diag}\left(a_{1}, \ldots, a_{n}\right)$, then one has

$$
E_{q}\left(A^{p+1} x ; A \theta\right)=\operatorname{diag}\left\{e_{q}\left(a_{1}^{p+1} x ; a_{1} \theta\right), \ldots, e_{q}\left(a_{n}^{p+1} x ; a_{n} \theta\right)\right\} .
$$


3. It satisfies $\mathcal{D} E_{q}\left(A^{p+1} x ; A \theta\right)=A E_{q}\left(A^{p+1} x ; A \theta\right)$.

4. It can also be written as $E_{q}\left(A^{p+1} x ; A \theta\right)=\sum_{k=0}^{p} \frac{(A \theta)^{k}}{[k]_{q} !} e^{A^{p+1} x}$.

Proof. The proof of the first property is clear. The second property is shown by a straightforward computation and the third property follows as in the scalar case by a direct calculation from the definitions. The last property follows since powers of $A$ commute.

Let us now consider the general linear system (6.1). Recalling the third property of $E_{q}$ stated in Proposition 6.1, it is clear that $w(x, \theta):=E_{q}\left(A^{p+1} x ; A \theta\right) c$ - where $c=\left(c_{1}, \ldots, c_{n}\right)^{t}$ with $c_{i} \in \mathbb{C}$ is a vector of constants - is a solution of (6.1). It remains to be shown that no other solutions exist. This will be done by an explicit construction of the solution.

Theorem 6.1. Let the linear system (6.1) with $A \in \mathbb{C}^{n, n}$ be given. Any solution $w(x, \theta)$ of $(6.1)$ can be written as

$$
w(x, \theta)=E_{q}\left(A^{p+1} x ; A \theta\right) c,
$$

where $c=\left(c_{1}, \ldots, c_{n}\right)^{t}$ with $c_{i} \in \mathbb{C}$ is a vector of constants. In particular, the set of solutions is a complex linear space of dimension $n$.

Proof. To show that all solutions can be written in this fashion we prove the asserted formula by constructing the solution. Inserting the function $w(x, \theta)=\left(w_{1}(x, \theta), \ldots, w_{n}(x, \theta)\right)^{t}$ into $(6.1)$ yields the following system

$$
\begin{aligned}
& \frac{1}{[p]_{q} !} \partial_{x} w_{i, 0}(x)=\sum_{l=1}^{n} a_{i l} w_{l, p}(x), \quad 1 \leq i \leq n, \\
& {[k]_{q} w_{i, k}(x)=\sum_{l=1}^{n} a_{i l} w_{l, k-1}(x), \quad 1 \leq i \leq n, \quad 1 \leq k \leq p,}
\end{aligned}
$$

which is equivalent to

$$
\begin{aligned}
& \left(\begin{array}{c}
\partial_{x} w_{1,0}(x) \\
\vdots \\
\partial_{x} w_{n, 0}(x)
\end{array}\right)=[p]_{q} ! A\left(\begin{array}{c}
w_{1, p}(x) \\
\vdots \\
w_{n, p}(x)
\end{array}\right), \\
& \left(\begin{array}{c}
w_{1, k}(x) \\
\vdots \\
w_{n, k}(x)
\end{array}\right)=\frac{A}{[k]_{q}}\left(\begin{array}{c}
w_{1, k-1}(x) \\
\vdots \\
w_{n, k-1}(x)
\end{array}\right), \quad 1 \leq k \leq p .
\end{aligned}
$$

A simple iteration shows that

$$
\left(\begin{array}{c}
w_{1, k}(x) \\
\vdots \\
w_{n, k}(x)
\end{array}\right)=\frac{A^{k}}{[k]_{q} !}\left(\begin{array}{c}
w_{1,0}(x) \\
\vdots \\
w_{n, 0}(x)
\end{array}\right), \quad 1 \leq k \leq p,
$$

and, consequently,

$$
\left(\begin{array}{c}
\partial_{x} w_{1,0}(x) \\
\vdots \\
\partial_{x} w_{n, 0}(x)
\end{array}\right)=A^{p+1}\left(\begin{array}{c}
w_{1,0}(x) \\
\vdots \\
w_{n, 0}(x)
\end{array}\right) .
$$


Thus, there exists a vector $c=\left(c_{1}, \ldots, c_{n}\right)^{t}$ of constants $c_{i} \in \mathbb{C}$ such that

$$
\left(\begin{array}{c}
w_{1,0}(x) \\
\vdots \\
w_{n, 0}(x)
\end{array}\right)=e^{A^{p+1} x}\left(\begin{array}{c}
c_{1} \\
\vdots \\
c_{n}
\end{array}\right)=e^{A^{p+1} x} c
$$

Collecting the above results shows that

$$
\begin{aligned}
w(x, \theta) & =\left(\begin{array}{c}
\sum_{k=0}^{p} w_{1, k}(x) \theta^{k} \\
\vdots \\
\sum_{k=0}^{p} w_{n, k}(x) \theta^{k}
\end{array}\right)=\sum_{k=0}^{p}\left(\begin{array}{c}
w_{1, k}(x) \\
\vdots \\
w_{n, k}(x)
\end{array}\right) \theta^{k}=\sum_{k=0}^{p} \frac{(A \theta)^{k}}{[k]_{q} !}\left(\begin{array}{c}
w_{1,0}(x) \\
\vdots \\
w_{n, 0}(x)
\end{array}\right) \\
& =\sum_{k=0}^{p} \frac{(A \theta)^{k}}{[k]_{q} !} e^{A^{p+1} x} c=E_{q}\left(A^{p+1} x ; A \theta\right) c,
\end{aligned}
$$

as asserted.

Remark 6.1. It is interesting to note that there is no simplification of the proof or the resulting formula for the case $p=1$ - in contrast to the scalar case where some simplifications occur.

\section{Some remarks concerning variable coefficients}

It is very important to note that we have here two types of variables, namely the ordinary "bosonic" one, i.e., $x$, and a nilpotent "fermionic" one, i.e., $\theta$. The fermionic variable is more an algebraic object then an analytical one, but here the two variables are mixed. Let us consider the linear differential equation with variable coefficients, i.e., an equation of the form

$$
\left(\mathcal{D}^{n}+c_{1}(x, \theta) \mathcal{D}^{n-1}+\cdots+c_{n-1}(x, \theta) \mathcal{D}+c_{n}(x, \theta)\right) f(x, \theta)=g(x, \theta),
$$

where the coefficients $c_{i}(c, \theta)$ are functions. We expect that it will be rather difficult to find solutions in general since the equation is more complex then the "ordinary" differential equation involving only the bosonic variable $x$. However, if we consider instead the coefficient functions to be depending only on the fermionic variable, i.e., $c_{i}(x, \theta) \equiv c_{i}(\theta)$, then the resulting differential equation

$$
\left(\mathcal{D}^{n}+c_{1}(\theta) \mathcal{D}^{n-1}+\cdots+c_{n-1}(\theta) \mathcal{D}+c_{n}(\theta)\right) f(x, \theta)=g(x, \theta)
$$

becomes more tractable since multiplication with the functions $c_{i}(\theta)$ results only in a swirling of the coefficients - and no "ordinary" differential equations with variable coefficients have to be solved as subproblem. Instead of discussing these equations further we illustrate them with one of the most simple examples.

Example 7.1. Let the differential equation

$$
(\mathcal{D}-c(\theta)) f(x, \theta)=0
$$

be given. Clearly, if $c(\theta)$ were constant, i.e., $c(\theta)=c$, then we would have to solve the eigenvalue equation $\mathcal{D} f(x, \theta)=c f(x, \theta)$ whose solution we have already found to be $f(x, \theta)=$ $C e_{q}\left(c^{p+1} x ; c \theta\right)$. Considering (7.1) and writing $c(\theta)=\sum_{l=0}^{p} c_{l} \theta^{l}$, the ansatz $f(x, \theta)=\sum_{k=0}^{p} f_{k}(x) \theta^{k}$ yields the system

$$
\frac{1}{[p]_{q} !} \partial_{x} f_{0}(x)=\sum_{l=0}^{p} c_{p-l} f_{l}(x), \quad[k+1]_{q} f_{k+1}(x)=\sum_{l=0}^{k} c_{k-l} f_{l}(x), \quad 0 \leq k \leq p-1 .
$$


The strategy to solve this equation is clear: Iteration of the second equation yields a relation of the form $f_{k}(x)=C_{k}(c(\theta), p) f_{0}(x)$ which can be inserted into the first equation to obtain a differential equation $\partial_{x} f_{0}(x)=D(c(\theta), p) f_{0}(x)$ which can be solved as $f_{0}(x)=C e^{D(c(\theta), p) x}$. Inserting this into the result for $f_{k}(x)$ yields then $f_{k}(x)$ and, consequently, $f(x, \theta)$. However, to determine the concrete values for the coefficients $C_{k}(c(\theta), p)$ and $D(c(\theta), p)$ seems to be difficult in the general case. As an illustration we consider the special case $p=2$. Here one obtains for $k=0$ the relation $f_{1}(x)=c_{0} f_{0}(x)$ and for $k=1$ the relation $[2]_{q} f_{2}(x)=c_{1} f_{0}(x)+c_{0} f_{1}(x)$, implying $f_{2}(x)=\frac{c_{1}+c_{0}^{2}}{[2]_{q}} f_{0}(x)$. These results can be inserted into the first equation and give

$$
\partial_{x} f_{0}(x)=\left\{c_{0}^{3}+\left(1+[2]_{q}\right) c_{0} c_{1}+[2]_{q} c_{2}\right\} f_{0}(x) \equiv D(c(\theta), 2) f_{0}(x) .
$$

Thus, $f_{0}(x)=C e^{D(c(\theta), 2) x}$. Inserting this into the equations for the components $f_{k}(x)$, we have finally found

$$
f(x, \theta)=C e^{D(c(\theta), 2) x}\left\{1+c_{0} \theta+\frac{c_{0}^{2}+c_{1}}{[2]_{q} !} \theta^{2}\right\} .
$$

Note that in the case where $c(\theta)$ is constant, i.e., $c(\theta)=c$, one has $D(c, 2)=c^{3}=c^{2+1}$ and the solution given above simplifies to $f(x, \theta)=C e^{c^{2+1} x}\left(1+c \theta+\frac{(c \theta)^{2}}{[2]_{q} !}\right)=C e_{q}\left(c^{2+1} x ; c \theta\right)$, as it should.

Remark 7.1. As noted already in the introduction, a certain differential equation involving a para-Grassmann variable was solved in [48] (by writing $f(x, \theta)=\sum_{k=0}^{p} f_{k}(x) \theta^{k}$, inserting this into the differential equation and comparing coefficients of $\theta^{k}$ ). Adapting the notation to the one used in the present paper, the differential equation considered in [48] can be written as

$$
\left[\partial_{x}+(\mu x+\nu) \sum_{l=0}^{p} \frac{(-\theta)^{l}}{l !} \partial_{x}^{l}\right] f(x, \theta)=\lambda f(x, \theta),
$$

where $\lambda, \mu, \nu \in \mathbb{C}$. Clearly, the structure of this differential equation differs from the structure of the differential equations considered in the present paper - see, e.g., equation (3.4) for a simple eigenvalue problem in our context.

\section{A simple family of nonlinear differential equations}

In the above sections we have considered certain types of linear differential equations and have found them to be very similar to "ordinary" differential equations (with only "bosonic" variables). In this section we want to discuss some equations which are closer to partial differential equations in "bosonic" variables. Let us start with the very simple nonlinear equation $(\mathcal{D} f(x, \theta))^{2}=0$. Solving this equation is straightforward: All one has to do is to insert (2.3) and compare coefficients. The result is that the general solution is given in the case $p=1,2$ by $f(x, \theta)=f_{0}(x)$ and in the general case of $p \geq 3$ by $f(x, \theta)=f_{0}(x)+\sum_{k=2+\left[\frac{p}{2}\right]}^{p} f_{k}(x) \theta^{k}$, where we have denoted by $[x]$ the largest integer smaller or equal than $x$. Thus, the general solution depends on $p-\left[\frac{p}{2}\right]$ functions. More generally, one may consider $\left(\mathcal{D}^{m} f(x, \theta)\right)^{n}=0$ for arbitrary $n \in \mathbb{N}$ and $m \leq p$. Writing $\mathcal{D}^{m} f(x, \theta) \equiv \sum_{l=0}^{p} h_{l}(x) \theta^{l}$ and considering $\left(\sum_{l=0}^{p} h_{l}(x) \theta^{l}\right)^{n}=0$ implies the vanishing of $h_{0}(x), \ldots, h_{\left[\frac{p}{n}\right]}(x)$. Applying (2.4) shows that $h_{0}(x)=f_{m}(x)$ and that the other $h_{j}(x)$ are given in a similar fashion where an interesting connection between $m, n$ and $p$ appears. These results are collected in the following theorem. 
Theorem 8.1. For arbitrary $p, n \in \mathbb{N}$ and $m \leq p$ the general solution of the differential equation $\left(\mathcal{D}^{m} f(x, \theta)\right)^{n}=0$ is given in the case $1 \leq m \leq p-\left[\frac{p}{n}\right]$ by

$$
f(x, \theta)=\sum_{k=0}^{m-1} f_{k}(x) \theta^{k}+\sum_{k=m+\left[\frac{p}{n}\right]+1}^{p} f_{k}(x) \theta^{k}
$$

and in the case $p-\left[\frac{p}{n}\right]<m \leq p$ by

$$
f(x, \theta)=\sum_{k=0}^{m-\left(p-\left[\frac{p}{n}\right]\right)-1} C_{k} \theta^{k}+\sum_{k=m-\left(p-\left[\frac{p}{n}\right]\right)}^{m-1} f_{k}(x) \theta^{k},
$$

where the appearing functions $f_{k}(x)$ are arbitrary (note that there are in both cases $p-\left[\frac{p}{n}\right]$ of them) and the $C_{k} \in \mathbb{C}$ are constants. Thus, the set of solutions has infinite dimensions.

Using the "periodicity" described in (2.5), the case where $m \geq p+1$ can be reduced to the one considered in the above theorem. In fact, writing $m=m^{\prime}(p+1)+m^{\prime \prime}$ with $0 \leq m^{\prime \prime} \leq p$, one has $\mathcal{D}^{m} f(x, \theta)=\mathcal{D}^{m^{\prime \prime}} h(x, \theta)$ with $h(x, \theta)=\partial_{x}^{m^{\prime}} f(x, \theta)$.

\section{Conclusions}

In this paper we have considered linear differential equations involving a para-Grassmann variable vanishing in $(p+1)$-th order. Suppose that we are given an equation of order $n=$ $n^{\prime}(p+1)+n^{\prime \prime}$. Expressing the parasuperfunction $f(x, \theta)$ through the components $f_{k}(x)$ and inserting this into the differential equation results in a system of $n^{\prime \prime}$ differential equations of order $n^{\prime}+1$ as well as $p-n^{\prime \prime}+1$ differential equations of order $n^{\prime}$ for the components $f_{k}(x)$. In the case $n \leq p$ one has, therefore, a system of $p-n+1$ recursion relations and $n$ differential equations of first order. The key to the solution of this system consists in expressing the "higher components" through the "lowest components" and then solving the resulting system of ordinary differential equations for the "lowest components". Having found the explicit form of the "lowest components" then allows one to describe the "higher components" and, consequently, $f(x, \theta)$ itself. For the linear differential equation with constant coefficients of order $n \leq p$ we have determined its solutions and found that the set of solutions is a complex linear space of dimension $n$ (the latter result also holds for arbitrary $n$ ). Characteristic for these linear differential equations with constant coefficients of order $n \leq p$ is an interesting blend of "ordinary" differential equations (involving only the "bosonic" variable $x$ ) and recursion relations. These recursion relations are intimately connected to the Fibonacci numbers and their generalizations to higher order. It is interesting to note that in the results there is no difference between the cases $p=1$ (i.e., an "ordinary" Grassmann variable) and $p>1$, although in the calculations (and proofs) there are striking differences: In the case $p=1$ the above-mentioned recursion relations are empty (i.e., not existent) and only a system of differential equations has to be solved. It also deserves to be mentioned that the appropriate exponential function is of utmost importance for describing the solutions. This is similar to the "bosonic" case but there exists a decisive difference: Here the "derivative" $\mathcal{D}$ is not a derivation, i.e., one does not have the usual law for the derivative of a product at hand. In particular, in the case corresponding to degenerated eigenvalues it is a priori not clear what the analogue of the usual ansatz $x^{n} e^{\lambda x}$ should be in the para-Grassmann case and one of the main difficulties met in the present work was to solve also these cases (even with hindsight - i.e., the formulas for the solution at hand it is not clear whether one would have made such an ansatz). Following the usual approach, the linear differential equation with constant coefficients can be transformed into a system 
of differential equations of first order. For such a general system we determined its solution and showed in particular that the set of solutions is also a complex linear space (of expected dimension).

We also considered some other classes of differential equations which, however, seem not as well behaved as the classes considered above and, therefore, deserve a closer study in the future. As a first example we mention the scalar linear differential equations where variable coefficients are allowed. We considered only an example of the case where the coefficients are functions of the para-Grassmann variable $\theta$ (no dependency on $x$ ). Again, one finds a blend of "ordinary" differential equations and recursion relations but due to the variable coefficients the components in the recursion relations obtain a "twist", resulting in slightly cumbersome expressions. It is obvious that for the general case some new ideas are needed. As a second example we considered a very simple family of nonlinear differential equations. Due to the nilpotency of the para-Grassmann variable $\theta$ we found a behavior than differs drastically from the linear case: Although the ususal blend of recursion relations and ordinary differential equations appears there exist (for appropriate choices of the involved parameters) several components of the solution for which no restrictions have to be satisfied. Thus, the dimension of the set of solutions is in general not $m$ (as one would have expected for the differential equation of order $m$ ) but infinite! Thus, the close analogy to the ordinary ("bosonic") differential equations found in the linear cases breaks down and one finds a behaviour which reminds one more of "bosonic" partial differential equations. Clearly, this is also a point where a closer study might reveal further interesting connections.

Before closing let us remark that - in addition to the above-mentioned points concerning differential equations involving one "bosonic" variable $x$ and one para-Grassmann variable $\theta-$ one can also consider partial differential equations involving several bosonic and para-Grassmann variables. Since in this case the para-Grassmann variables are - in addition to being nilpotent noncommutative, it is to be expected that it will be much harder to obtain results for these paraGrassmann partial differential equations than for the "ordinary" para-Grassmann differential equations considered in the present paper. Before beginning this journey into the unknown territory full of treasures one should prepare oneself to meet surprises - pleasant or otherwise around every corner and it seems mandatory to become comfortable with those of them showing up already in the one-variable case. This is what we began in the present paper.

\section{Acknowledgements}

The authors would like to thank V.I. Tkach and R.M. Yamaleev for instructive correspondence as well as the anonymous referees for several suggestions improving the paper.

\section{References}

[1] Schwinger J., The theory of quantized fields. IV, Phys. Rev. 92 (1953), 1283-1299.

[2] Martin J.L., The Feynman principle for a Fermi system, Proc. Roy. Soc. London. Ser. A 251 (1959), 543-549.

[3] Berezin F., The method of second quantization, Pure and Applied Physics, Vol. 24, Academic Press, New York - London, 1966.

[4] Kane G., Shifman M. (Editors), The supersymmetric world. The beginnings of the theory, World Scientific Publishing Co., Inc., River Edge, NJ, 2000.

[5] Wess J., Bagger J., Supersymmetry and supergravity, 2nd ed., Princeton Series in Physics, Princeton University Press, Princeton, 1992.

[6] Freund P., Introduction to supersymmetry and supergravity, 2nd ed., World Scientific Publishing Co., Inc., Teaneck, NJ, 1990.

[7] DeWitt B., Supermanifolds, 2nd ed., Cambridge Monographs on Mathematical Physics, Cambridge University Press, Cambridge, 1992. 
[8] Manin Y.I., Gauge field theory and complex geometry, Springer-Verlag, Berlin, 1988.

[9] Rogers A., Supermanifolds. Theory and applications, World Scientific Publishing Co. Pte. Ltd., Hackensack, NJ, 2007.

[10] Green H.S., A generalized method of field quantization, Phys. Rev. 90 (1953), 270-273.

[11] Kalnay A.J., A note on Grassmann algebras, Rep. Math. Phys. 9 (1976), 9-13.

[12] Omote M., Kamefuchi S., Para-Grassmann algebras and para-Fermi systems, Lett. Nuovo Cimento 24 (1979), 345-350.

[13] Omote M., Kamefuchi S., Parafields and supergroup transformations, Nuovo Cimento A 50 (1980), 21-40.

[14] Ohnuki Y., Kamefuchi S., Para-Grassmann algebras with applications to para-Fermi systems, J. Math. Phys. 21 (1980), 609-616.

[15] Ohnuki Y., Kamefuchi S., Quantum field theory and parastatistics, Springer-Verlag, Berlin, 1982.

[16] Gershun V.D., Tkach V.I., Para-Grassmann variables and description of massive particles with spin equalling one, Ukr. Fiz. Zh. 29 (1984), 1620-1627 (in Russian).

[17] Zheltukhin A.A., Para-Grassmann extension of the Neveu-Schwarz-Ramond algebra, Theoret. and Math. Phys. 71 (1987), 491-496.

[18] Rubakov V.A., Spirodonov V.P., Parasupersymmetric quantum mechanics, Modern Phys. Lett. A 3 (1988), $1337-1347$.

[19] Yamaleev R.M., Elements of cubic quantum mechanics, JINR Comm. P2-88-147, 1988 (in Russian).

[20] Durand S., Floreanini R., Mayrand M., Vinet L., Conformal parasuperalgebras and their realizations on the line, Phys. Lett. B 233 (1989), 158-162.

[21] Durand S., Mayrand M., Spirodonov V.P., Vinet L., Higher order parasupersymmetric quantum mechanics, Modern Phys. Lett. A 6 (1991), 3163-3170.

[22] Fleury N., Rausch de Traubenberg M., Yamaleev R.M., Matricial representations of rational powers of operators and para-Grassmann extension of quantum mechanics, Internat. J. Modern Phys. A 10 (1995), 1269-1280.

[23] Yamaleev R.M., Fractional power of momenta and para-Grassmann extension of Pauli equation, Adv. Appl. Clifford Algebras 7 (1997), suppl., 279-288.

[24] Yamaleev R.M., Parafermionic extensions of Pauli and Dirac equations, Hadronic J. 26 (2003), $247-258$.

[25] Durand S., Factional supersymmetry and quantum mechanics, Phys. Lett. B 312 (1993), 115-120, hep-th/9305128.

[26] Durand S., Extended fractional supersymmetric quantum mechanics, Modern Phys. Lett. A 8 (1993), 17951804, hep-th/9305129.

[27] Durand S., Fractional superspace formulation of generalized mechanics, Modern Phys. Lett. A 8 (1993), 2323-2334, hep-th/9305130.

[28] Azcárraga J.A., Macfarlane A.J., Group theoretical foundations of fractional supersymmetry, J. Math. Phys. 37 (1996), 1115-1127, hep-th/9506177.

[29] Dunne R.S., Macfarlane A.J., de Azcárraga J.A., Pérez Bueno J.C., Geometrical foundations of fractional supersymmetry, Internat. J. Modern Phys. A 12 (1997), 3275-3305, hep-th/9610087.

[30] Ahn C., Bernard D., LeClair A., Fractional supersymmetries in perturbed coset CFTs and integrable soliton theory, Nuclear Phys. B 346 (1990), 409-439.

[31] Saidi E.H., Sedra M.B., Zerouaoui J., On $D=2(1 / 3,1 / 3)$ supersymmetric theories. I, Classical Quantum Gravity 12 (1995), 1567-1580.

[32] Saidi E.H., Sedra M.B., Zerouaoui J., On $D=2(1 / 3,1 / 3)$ supersymmetric theories. II, Classical Quantum Gravity 12 (1995), 2705-2714.

[33] Fleury N., Rausch de Traubenberg M., Local fractional supersymmetry for alternative statistics, Modern Phys. Lett. A 11 (1996), 899-913, hep-th/9510108.

[34] Perez A., Rausch de Traubenberg M., Simon P., 2D-fractional supersymmetry and conformal field theory for alternative statistics, Nuclear Phys. B 482 (1996), 325-344, hep-th/9603149.

[35] Rausch de Traubenberg M., Simon P., 2D-fractional supersymmetry and conformal field theory for alternative statistics, Nuclear Phys. B 517 (1997), 485-505, hep-th/9606188. 
[36] Kheirandish F., Khorrami M., Two-dimensional fractional supersymmetric conformal field theories and the two point functions, Internat. J. Modern Phys. A 16 (2001), 2165-2173, hep-th/0004154.

[37] Kheirandish F., Khorrami M., Logarithmic two-dimensional spin 1/3 fractional supersymmetric conformal field theories and the two point functions, Eur. Phys. J. C Part. Fields 18 (2001), 795-797, hep-th/0007013.

[38] Kheirandish F., Khorrami M., Two-dimensional fractional supersymmetric conformal and logarithmic conformal field theories and the two point functions, Eur. Phys. J. C Part. Fields 20 (2001), 593-597, hep-th/0007073.

[39] Sedra M.B., Zerouaoui J., Heterotic $D=2(1 / 3,0)$ SUSY models, arXiv:0903.1316.

[40] Durand S., Fractional superspace formulation of generalized super-Virasoro algebras, Modern Phys. Lett. A 7 (1992), 2905-2911, hep-th/9205086.

[41] Filippov A.T., Isaev A.P., Kurdikov A.B., Para-Grassmann extensions of the Virasoro algebra, Internat. J. Modern Phys. A 8 (1993), 4973-5003, hep-th/9212157.

[42] Filippov A.T., Isaev A.P., Kurdikov A.B., Para-Grassmann analysis and quantum groups, Modern Phys. Lett. A 7 (1992), 2129-2141, hep-th/9204089.

[43] Rausch de Traubenberg M., Clifford algebras of polynomials, generalized Grassmann algebras and $q$ deformed Heisenberg algebras, Adv. Appl. Clifford Algebras 4 (1994), 131-144, hep-th/9404057.

[44] Abdesselam B., Beckers J., Chakrabarti A., Debergh N., On a deformation of sl(2) with para-Grassmannian variables, J. Phys. A: Math. Gen. 29 (1996), 6729-6736, q-alg/9507008.

[45] Isaev A.P., Para-Grassmann integral, discrete systems and quantum groups, Internat. J. Modern Phys. A 12 (1997), 201-206, q-alg/9609030.

[46] Plyushchay M.A., R-deformed Heisenberg algebra, Modern Phys. Lett. A 11 (1996), 2953-2964, hep-th/9701065.

[47] Plyushchay M.A., Deformed Heisenberg algebra with reflection, Nuclear Phys. B 491 (1997), 619-634, hep-th/9701091.

[48] Alvarez-Moraga N., Coherent and squeezed states of quantum Heisenberg algebras, J. Phys. A: Math. Gen. 38 (2005), 2375-2398, math-ph/0503055.

[49] Cabra D.C., Moreno E.F., Tanasa A., Para-Grassmann variables and coherent states, SIGMA 2 (2006), 087, 8 pages, hep-th/0609217.

[50] Rausch de Traubenberg M., Fleury N., Beyond spinors, in Leite Lopes Festschrift, Editors N. Fleury et al., World Scientific Publishing Co., Singapore, 1988, 79-101.

[51] Filippov A.T., Isaev A.P., Kurdikov A.B., Para-Grassmann differential calculus, Theoret. and Math. Phys. 94 (1993), 150-165, hep-th/9210075.

[52] Cugliandolo L.F., Lozano G.S., Moreno E.F., Schaposnik F.A., A note on Gaussian integrals over paraGrassmann variables, Internat. J. Modern Phys. A 19 (2004), 1705-1714, hep-th/0209172.

[53] Schork M., Algebraical, combinatorial and analytical properties of paragrassmann variables, Internat. J. Modern Phys. A 20 (2005), 4797-4819.

[54] Rausch de Traubenberg M., Clifford algebras, supersymmetry and $Z(n)$ symmetries: applications in field theory, hep-th/9802141.

[55] van der Put M., Singer M.F., Galois theory of linear differential equations, Springer-Verlag, Berlin, 2003.

[56] Miles E.P., Generalized Fibonacci numbers and associated matrices, Amer. Math. Monthly 67 (1960), 745752 .

[57] Levesque C., On $m$ th order linear recurrences, Fibonacci Quart. 23 (1985), 290-293.

[58] Lee G.-Y., Lee S.-G., Kim J.-S., Shin H.-G., The Binet formula and representations of $k$-generalized Fibonacci numbers, Fibonacci Quart. 39 (2001), 158-164. 